Article

\title{
Effects of Olive Oil on Markers of Inflammation and Endothelial Function-A Systematic Review and Meta-Analysis
}

\author{
Lukas Schwingshackl ${ }^{1,2}$, Marina Christoph ${ }^{1}$ and Georg Hoffmann ${ }^{1, *}$ \\ ${ }^{1}$ Department of Nutritional Sciences, Faculty of Life Sciences, University of Vienna, \\ Athanstraße 14 (UZAII), Vienna A-1090, Austria; E-Mails: lukas.schwingshackl@ univie.ac.at (L.S.); \\ a0902438@unet.univie.ac.at (M.C.) \\ 2 Department of Epidemiology, German Institute of Human Nutrition Potsdam-Rehbruecke (DIfE), \\ Arthur-Scheunert-Allee 114-116, 14558 Nuthetal, Germany \\ * Author to whom correspondence should be addressed; E-Mail: georg.hoffmann@univie.ac.at; \\ Tel.: +43-1-4277-54950; Fax: +43-1-4277-9549.
}

Received: 27 May 2015 / Accepted: 6 September 2015 / Published: 11 September 2015

\begin{abstract}
The aim of the present systematic review was to synthesize data from randomized controlled trials investigating the effects of olive oil on markers of inflammation or endothelial function. Literature search in electronic databases Cochrane Trial Register, EMBASE, and MEDLINE was performed. Thirty studies enrolling 3106 participants fulfilled the selection criteria. Pooled effects of different interventions were assessed as mean difference using a random effects model. Olive oil interventions (with daily consumption ranging approximately between $1 \mathrm{mg}$ and $50 \mathrm{mg}$ ) resulted in a significantly more pronounced decrease in $C$-reactive protein (mean difference: $-0.64 \mathrm{mg} / \mathrm{L},(95 \%$ confidence interval (CI) -0.96 to -0.31$), p<0.0001, n=15$ trials) and interleukin-6 (mean difference: -0.29 (95\% CI -0.7 to -0.02$), p<0.04, n=7$ trials) as compared to controls, respectively. Values of flow-mediated dilatation (given as absolute percentage) were significantly more increased in individuals subjected to olive oil interventions (mean difference: $0.76 \%$ (95\% CI 0.27 to 1.24$), p<0.002, n=8$ trials). These results provide evidence that olive oil might exert beneficial effects on endothelial function as well as markers of inflammation and endothelial function, thus representing a key ingredient contributing to the cardiovascular-protective effects of a Mediterranean diet. However, due to the heterogeneous study designs (e.g., olive oil given as a supplement or as part of dietary pattern, variations in control diets), a conservative interpretation of the results is necessary.
\end{abstract}


Keywords: cardiovascular disease; $C$-reactive protein; flow-mediated dilatation; interleukin-6; Mediterranean diet

\section{Introduction}

Cardiovascular diseases (CVD) represent the main cause of disability and death in industrialized countries. Numerous risk factors are known to promote the pathogenesis of CVD. Some of these risk factors are modifiable lifestyle habits such as physical activity and choice of foods. Diets low in fruits, vegetables, nuts and seeds, whole grains, or $n-3$ rich seafood but high in sodium, salt, and sugar-sweetened beverages are regarded to increase the risk of CVD [1]. Thus, the lower incidence rates of CVD observed in Southern Europe might at least in part be explained by health-promoting dietary patterns such as the Mediterranean diet (MedD) rich in extra virgin olive oil, fruits, vegetables, nuts and seeds, legumes, but low in red meat and dairy products [2]. Extra virgin olive oil is the main source of fat in the MedD. With its high content in monounsaturated fatty acids and polyphenols, extra virgin olive oil might exert beneficial effects in the development and progression of diseases associated with chronic low-grade inflammation [3-5].

Low-grade, chronic synthesis and release of pro-inflammatory cytokines like interleukin-1 (IL-1) and tumor necrosis factor- $\alpha$ (TNF- $\alpha$ ) within the vascular wall affect endothelial function e.g., via up-regulation of adhesion molecules [6]. Over time, this may lead to endothelial dysfunction, a predictor as well as a pathogenic mechanism of atherosclerosis and CVD [7]. Thus, excessive inflammatory processes of the endothelium were reported to be predictors of future cardiovascular events [8]. In a recent systematic review of randomized controlled studies (RCT) we showed that adherence to a MedD was associated with improvements in endothelial function as well as inflammatory status [9]. Beneficial effects of an MedD on markers of both inflammation and endothelial function have been reported by others as well [10-12]. One potential component mediating the positive effects of a MedD might be the prominent role of olive oil in this dietary pattern. Bioactive components of extra virgin olive oil have demonstrated endothelium-protective and anti-oxidative properties [4,13]. Therefore, consumption of extra virgin olive oil might explain the beneficial effects of a MedD with respect to incidences of CVD. To gain additional information about the effects of olive oil (a major component of the MedD) on parameters involved in the development of CVD, it was the aim of the present systematic review to synthesize data of randomized controlled trials investigating the effects of olive oil interventions (administered either in the form of capsules, supplemented to the habitual diet, or supplemented to a specific dietary pattern such as MedD) on markers of inflammation as well as endothelial function.

\section{Experimental Section}

This systematic review was registered in the PROSPERO International Prospective Register of Systematic Reviews [14], indexed under CRD42014008803. 


\subsection{Literature Search}

Literature search was conducted using the electronic databases PubMed (1966 until June 2015), EMBASE (1980 until June 2015) and the Cochrane Trial Register (until June 2015). The following search terms were used: ("olive oil") and ("endothelial" or "inflammation" or "CRP" or " $C$-reactive protein" or "FMD" or "flow-mediated dilatation") and ("randomized controlled trial" or "randomized" or "clinical trials as topic" or "placebo" or "randomly" or "trial") not ("animals"). Reference lists of retrieved articles, systematic reviews, and meta-analyses were checked for further relevant trials.

\subsection{Study Selection}

The following inclusion criteria were defined prior to study selection process:

(1) intervention with olive oil in pure form or as supplement (capsules); (2) randomized controlled trials (RCT) with either parallel or crossover design; (3) participants $\geqslant 19$ years of age; (4) minimum intervention period of four weeks; (5) no other supplementation; (6) assessment of the "outcome of interest": Markers of inflammation ( $C$-reactive protein (CRP), interleukin-6 (IL-6), TNF- $\alpha$, adiponectin) and endothelial function (intercellular adhesion molecule-1 (ICAM-1), vascular cell adhesion molecule-1 (VCAM-1), flow-mediated dilatation (FMD)); (7) report of post-intervention mean values (or if not available, change from baseline values were used instead) with standard deviation (or basic data which allow to calculate these parameters, i.e., standard errors, 95\% confidence interval, $p$-values).

\subsection{Risk of Bias Assessment}

The Cochrane Collaboration's tool for assessing risk of bias was used to elucidate the risk of bias of the included studies attaching either low, unclear or high risk of bias to the seven domains (sequence generation, allocation concealment, blinding of participants and personnel, blinding of outcome assessment, incomplete outcome data, selective outcome reporting) to each study [15].

\subsection{Data Extraction and Analyses}

Data extracted from each trial were: first author's family name; year of publication; number, age, and gender distribution of study participants; duration of intervention; description of olive oil intervention and respective control; outcomes and post-intervention mean values or differences in mean of two time points together with corresponding standard deviations.

All data were analyzed using the software REVIEW MANAGER 5.3. as provided by the Cochrane Collaboration [16]. In a random effects model, the post-mean values or the changes from baseline values and corresponding standard deviations of intervention and control/intervention groups were compared. Pooled effects of the different interventions were investigated as mean difference. Heterogeneity between trial results was tested with a standard $\chi^{2}$ test. The $I^{2}$ parameter was used to quantify any inconsistency: $I^{2}=((\mathrm{Q}-\mathrm{d} f)) / \mathrm{Q} \times 100 \%$, where $\mathrm{Q}$ is the $\chi^{2}$ statistic and $\mathrm{d} f$ is its degrees of freedom. An $I^{2}$-value of greater than $50 \%$ was considered to represent considerable heterogeneity.

Literature search was performed by Lukas Schwingshackl and Marina Christoph, while data extraction, analyses, and synthesis was done by all authors with disagreement resolved by consensus. 


\subsection{Specific Data Handling/Handling of Missing Data}

After contacting the corresponding authors, the missing data of the trial by Damasceno et al. [17] as well as the CRP values of the trial by Sanders et al. [16] could be added to the meta-analysis.

\section{Results}

\subsection{Literature Search and Study Characteristics}

Selection criteria were fulfilled by 30 studies with altogether 3106 participants [4,17-46]. The detailed steps of the selection process is summarized in Figure 1. Figure 2 summarizes the distribution of the risk of bias for all domains and all studies.

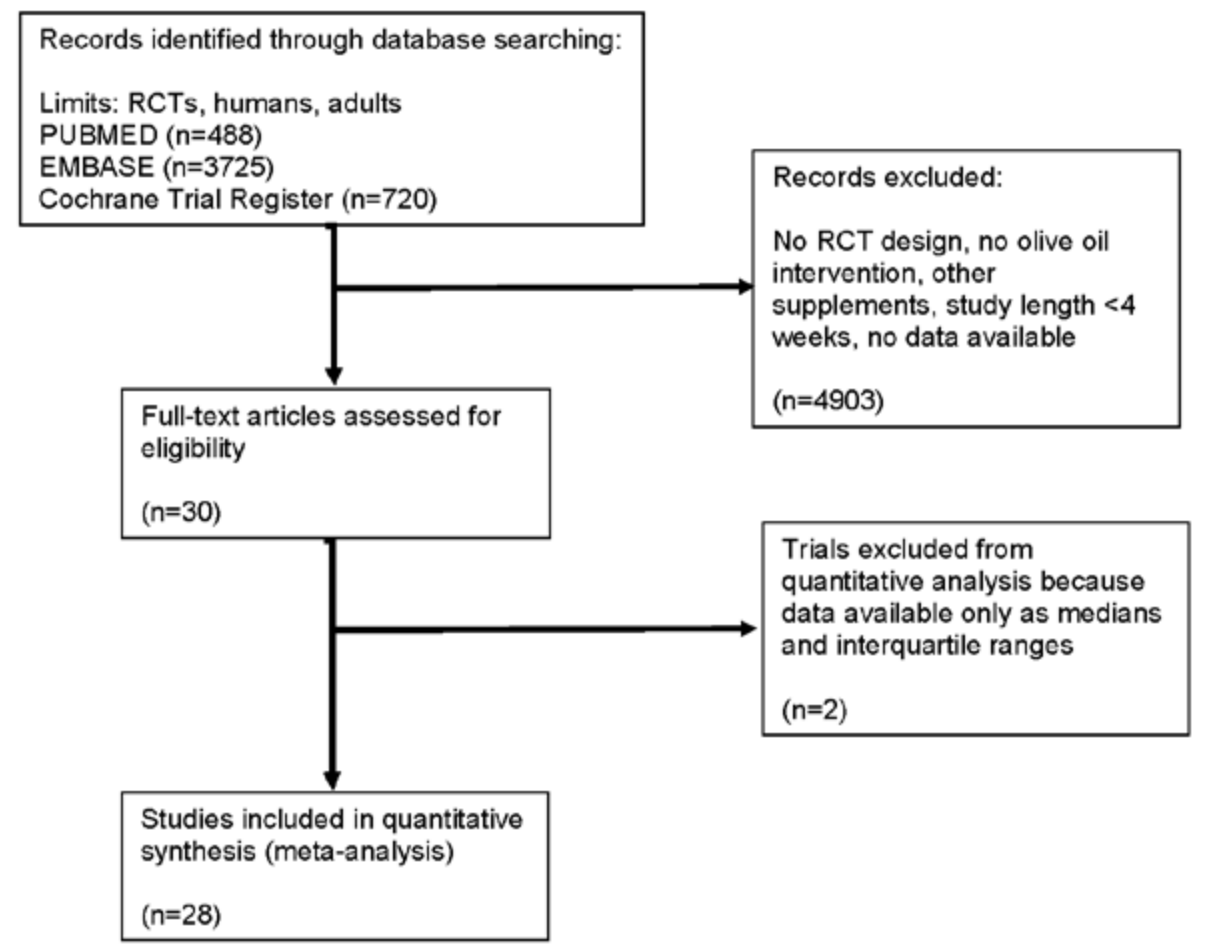

Figure 1. Flow diagram.

Random sequence generation (selection bias)

Allocation concealment (selection bias)

Blinding of participants and personnel (performance bias)

Blinding of outcome assessment (detection bias)
Incomplete outcome data (attrition bias)
Selective reporting (reporting bias)

Blinding of outcome assessment (detection bias)
Incomplete outcome data (attrition bias)
Selective reporting (reporting bias)

Blinding of outcome assessment (detection bias)
Incomplete outcome data (attrition bias)
Selective reporting (reporting bias)
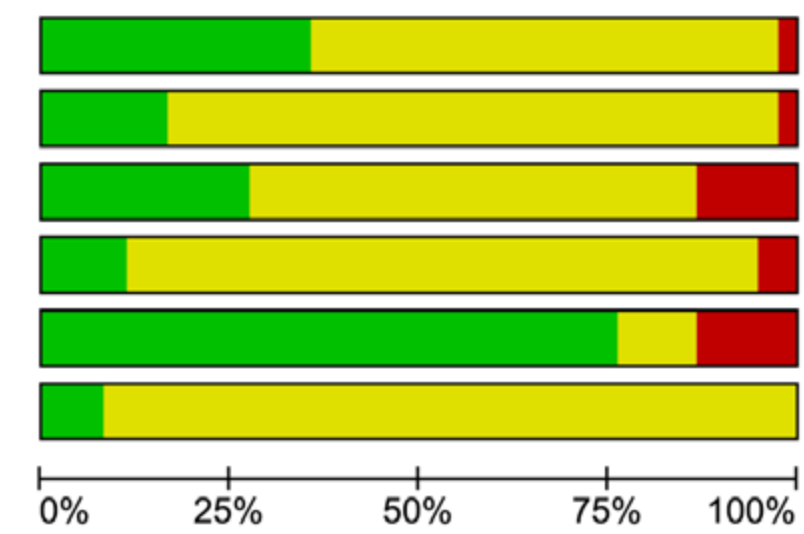


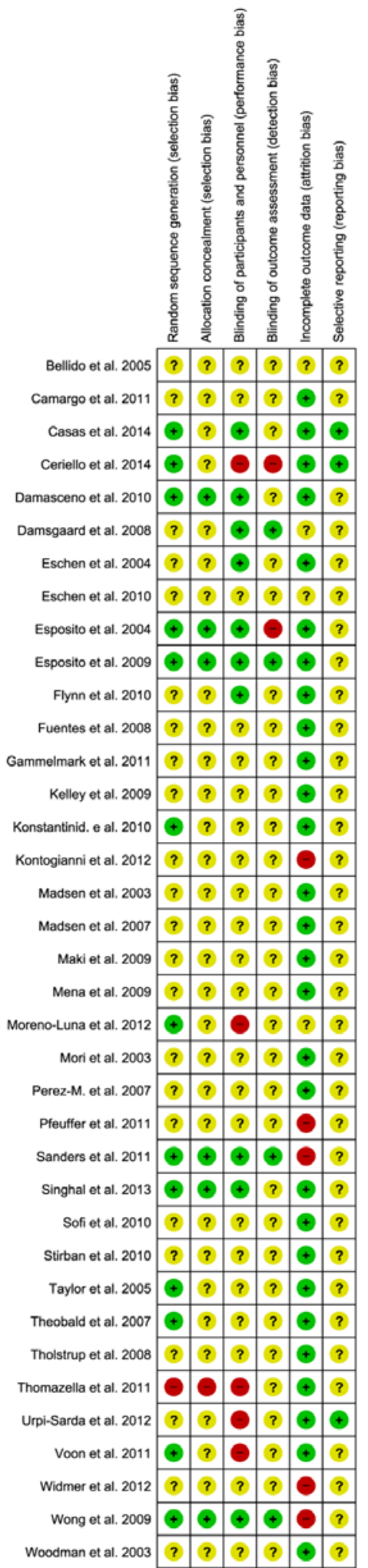

Figure 2. Risk of bias assessment tool. (A) Across trials, information is either from trials at low risk of bias (green), or from trials at unclear risk of bias (yellow), or from trials at high risk of bias (red). (B) For each study, every bias domain will be checked, the given summary represents an assessment of bias risk across studies. For each bias domain, low risk of bias means that information is from studies at low risk of bias (green), high risk of bias indicates the proportion of information from studies at high risk of bias which might be sufficient to affect the interpretation of the results (red), and unclear risk of bias refers to information from studies at low or unclear risk of bias (yellow). 
The study duration varied between four and 208 weeks. Part of the RCTs was comparing MedD supplemented with (virgin/extra virgin) olive oil and a control diet. Another subset of RCTs compared olive oil capsules against capsules containing other fats. Due to the different designs of the different interventions, the studies were classified in subgroups according to mode of olive oil intervention and controls as follows:

A Mediterranean diet (MedD) with a specified quantity of olive oil vs.

(1) low-fat diet;

(2) healthy diet;

(3) MedD and nuts;

(4) Western diet/diet high in carbohydrates;

A specified quantity of olive oil supplemented to the habitual diet $v s$.

(1) flaxseed oil;

(2) coconut oil/palm oil;

(3) olive oil with $n-3$ fatty acids;

A specified quantity of olive oil in capsules supplemented to the habitual diet $v s$.

(1) conjugated linoleic acid mix capsules;

(2) n-3 fatty acid capsules;

Or specific comparisons between plant-based diets with other dietary patterns, i.e.,

(1) plant-based olive oil diet vs. National Cancer Institute diet;

(2) monounsaturated fatty acid diet $v s$. saturated fatty acid diet and low-fat diet.

General study characteristics are summarized in Table 1. 
Table 1. General characteristics of randomized controlled trials included in the systematic review.

\begin{tabular}{|c|c|c|c|c|c|c|c|c|}
\hline Reference & $\begin{array}{c}\text { Sample } \\
\text { Size; } \\
\text { \% Female }\end{array}$ & $\begin{array}{c}\text { Age } \\
(\text { Years, Mean } \pm \text { SD) }\end{array}$ & $\begin{array}{c}\text { BMI } \\
\left(\mathrm{kg} / \mathrm{m}^{2}, \text { Mean } \pm \mathrm{SD}\right)\end{array}$ & $\begin{array}{l}\text { Duration } \\
\text { (Weeks), } \\
\text { Design }\end{array}$ & $\begin{array}{c}\text { Diseases/Health } \\
\text { Status/Medication }\end{array}$ & $\begin{array}{c}\text { Olive Oil } \\
\text { Intervention }\end{array}$ & Comparison Group & $\begin{array}{l}\text { Outcome } \\
\text { Parameters }\end{array}$ \\
\hline $\begin{array}{l}\text { Casas et al., } \\
2014 \text { [19] }\end{array}$ & $\begin{array}{l}164 ; \\
\text { olive oil: } \\
56.4 \\
\text { control: } \\
59.3\end{array}$ & $\begin{array}{c}\text { Olive oil: } 68.1 \pm 6 \\
\text { control: } 67.4 \pm 4\end{array}$ & $\begin{array}{c}\text { Olive oil: } 27.9 \pm 3.4, \\
\text { control: } 26.5 \pm 3.7\end{array}$ & 52 , parallel & $\begin{array}{l}\text { High risk for CVD } \\
\text { (type } 2 \text { diabetes } \\
\text { mellitus or } 3 \text { or more } \\
\text { major cardiovascular } \\
\text { risk factors) }\end{array}$ & $\begin{array}{c}\text { MedD + extra } \\
\text { virgin olive oil } \\
(50 \mathrm{~mL} / \text { day })\end{array}$ & Low-fat diet & $\begin{array}{c}\text { CRP, } \\
\text { sE-selectin, } \\
\text { sP-selectin, } \\
\text { sVCAM-1 }\end{array}$ \\
\hline $\begin{array}{l}\text { Ceriello et al., } \\
2014 \text { [20] }\end{array}$ & $\begin{array}{l}24 ; \\
\text { olive oil: } 25 \text {, } \\
\text { control: } 33\end{array}$ & n.d. & $\begin{array}{c}\text { Olive oil: } 29.8 \pm 1.4, \\
\text { control: } 29.2 \pm 1.1\end{array}$ & $\begin{array}{l}3 \text { months, } \\
\text { parallel }\end{array}$ & $\begin{array}{l}\text { Type } 2 \text { diabetes } \\
\text { mellitus }\end{array}$ & $\begin{array}{c}\text { MedD + extra } \\
\text { virgin olive oil } \\
(50 \mathrm{~mL} / \text { day })\end{array}$ & Low-fat diet & FMD \\
\hline $\begin{array}{l}\text { Damasceno et al., } \\
2010 \text { [17] }\end{array}$ & $18 ; 50$ & $56 \pm 13$ & $25.7 \pm 2.3$ & $\begin{array}{c}4, \\
\text { cross-over }\end{array}$ & $\begin{array}{l}\text { Moderate } \\
\text { hypercholesterolemia, } \\
\text { no medication, no } \\
\text { hormone replacement } \\
\text { therapy }\end{array}$ & $\begin{array}{l}\text { MedD + 30-50 } \\
\text { g/day virgin } \\
\text { olive oil } \\
\text { (polyphenols } \\
34.3 \mathrm{mg} / 100 \mathrm{~g} \text { ) }\end{array}$ & $\begin{array}{l}\text { MedD + walnuts } \\
\text { (polyphenols } 1.3 \\
\text { mg/100g); } \\
\text { MedD + almonds } \\
\text { (polyphenols } 1.1 \\
\text { mg/100g) }\end{array}$ & $\begin{array}{c}\text { CRP, } \\
\text { sICAM-1, } \\
\text { sVCAM-1 }\end{array}$ \\
\hline $\begin{array}{l}\text { Damsgaard et al., } \\
2008 \text { [21] }\end{array}$ & $\begin{array}{c}64 \\
0\end{array}$ & $\begin{array}{l}\text { Olive oil high linoleic } \\
\text { acid: } 24.9 \pm 3.8, \\
\text { olive oil low linoleic } \\
\text { acid: } 25.5 \pm 4.4, \\
\text { control high linoleic } \\
\text { acid: } 26.3 \pm 4.8, \\
\text { control low linoleic } \\
\text { acid: } 24.9 \pm 4.9\end{array}$ & $\begin{array}{l}\text { Olive oil high linoleic } \\
\text { acid: } 23.1 \pm 1.9, \\
\text { olive oil low linoleic } \\
\text { acid: } 23.3 . \pm 1.9, \\
\text { control high linoleic } \\
\text { acid: } 21.9 \pm 1.8, \\
\text { control low linoleic } \\
\text { acid: } 22.9 \pm 1.9\end{array}$ & $\begin{array}{c}8 \\
2 \times 2 \\
\text { factorial }\end{array}$ & $\begin{array}{l}\text { Healthy, no } \\
\text { medication }\end{array}$ & $\begin{array}{l}5 \mathrm{~mL} / \text { day } \\
\text { unrefined extra } \\
\text { virgin olive oil } \\
\text { capsules }\end{array}$ & $\begin{array}{c}5 \mathrm{~mL} / \text { day fish oil } \\
\text { capsules }\end{array}$ & $\begin{array}{l}\text { adiponectin, } \\
\text { CRP, IL-6, } \\
\text { VCAM-1 }\end{array}$ \\
\hline
\end{tabular}


Table 1. Cont.

\begin{tabular}{|c|c|c|c|c|c|c|c|c|}
\hline $\begin{array}{l}\text { Damsgaard et al., } \\
2009 \text { [22] }\end{array}$ & $\begin{array}{c}63 \\
0\end{array}$ & $\begin{array}{l}\text { Olive oil high linoleic } \\
\text { acid: } 24.9 \pm 3.8, \\
\text { olive oil low linoleic } \\
\text { acid: } 25.5 \pm 4.4, \\
\text { control high linoleic } \\
\text { acid: } 26.3 \pm 4.8, \\
\text { control low linoleic } \\
\text { acid: } 24.9 \pm 4.9\end{array}$ & $\begin{array}{c}\text { Olive oil high linoleic } \\
\text { acid: } 23.1 \pm 1.9, \\
\text { olive oil low linoleic } \\
\text { acid: } 23.3 . \pm 1.9 \\
\text { control high linoleic } \\
\text { acid: } 21.9 \pm 1.8 \\
\text { control low linoleic } \\
\text { acid: } 22.9 \pm 1.9\end{array}$ & $\begin{array}{c}8 \\
2 \times 2 \\
\text { factorial }\end{array}$ & $\begin{array}{l}\text { Healthy, no intensive } \\
\text { sports }\end{array}$ & $\begin{array}{l}\quad 5 \mathrm{~mL} / \text { day } \\
\text { unrefined extra } \\
\text { virgin olive oil } \\
\quad \text { capsules }\end{array}$ & $\begin{array}{c}5 \mathrm{~mL} / \text { day fish oil } \\
\text { capsules }\end{array}$ & \\
\hline $\begin{array}{c}\text { Eschen } \text { et al., } \\
2004 \text { [23] }\end{array}$ & $\begin{array}{c}60 ; \\
\text { olive oil: } 82 \text {, } \\
\text { control: } 66\end{array}$ & $\begin{array}{l}\text { Olive oil: } 39 \pm 10 \\
\text { control: } 38 \pm 11\end{array}$ & $\begin{array}{c}\text { Olive oil: } 24.6 \pm 3.7 \\
\text { control: } 25.1 \pm 2.9\end{array}$ & 12 , parallel & $\begin{array}{l}\text { Healthy, no } \\
\text { medication }\end{array}$ & $\begin{array}{c}10 \text { g/day olive } \\
\text { oil capsules }\end{array}$ & $\begin{array}{c}6.6 \text { g/day } n \text {-3 PUFA } \\
\text { capsules; } 2.0 \text { g/day } n-3 \\
\text { PUFA + } 7 \text { g/day olive } \\
\text { oil capsules }\end{array}$ & $\begin{array}{l}\text { ICAM-1, } \\
\text { VCAM-1, } \\
\text { P-selectin }\end{array}$ \\
\hline $\begin{array}{c}\text { Eschen et al., } \\
2010 \text { [24] }\end{array}$ & $\begin{array}{l}\text { 138; } \\
\text { olive oil: } 22 \text {, } \\
\text { control: } 13\end{array}$ & $\begin{array}{l}\text { Olive oil: } 61 \pm 8 \\
\text { control: } 58 \pm 10\end{array}$ & $\begin{array}{c}\text { Olive oil: } 27.1 \pm 0.4 \\
\text { control: } 27.1 \pm 0.5\end{array}$ & 24 , parallel & $\begin{array}{l}\text { Chronic heart failure } \\
\text { and left ventricular } \\
\text { ejection fraction }<40 \\
\text { or stable oral } \\
\text { medication }\end{array}$ & $\begin{array}{l}1 \mathrm{~g} / \text { day olive oil } \\
\text { capsules }\end{array}$ & $\begin{array}{c}0.9 \text { g/day } n-3 \text { PUFA } \\
\text { capsules }\end{array}$ & $\begin{array}{l}\text { SICAM-1, } \\
\text { sVCAM-1, } \\
\text { sP-selectin }\end{array}$ \\
\hline $\begin{array}{c}\text { Esposito et al., } \\
2004 \text { [25] }\end{array}$ & $\begin{array}{c}\text { 180; } \\
\text { olive oil: } 46 \text {, } \\
\text { control: } 44\end{array}$ & $\begin{array}{c}\text { Olive oil: } 44.3 \pm 6.4 \\
\text { control: } 43.5 \pm 5.9\end{array}$ & $\begin{array}{c}\text { Olive oil: } 27.9 \pm 3.4 \\
\text { control: } 28.1 \pm 3.2\end{array}$ & $\begin{array}{c}104, \\
\text { parallel }\end{array}$ & $\begin{array}{l}\geqslant 3 \text { criteria of } \\
\text { metabolic syndrome, } \\
\text { no medication, no } \\
\text { intensive sports }\end{array}$ & $\begin{array}{c}\text { MedD + olive } \\
\text { oil }\end{array}$ & Generally healthy diet & $\begin{array}{l}\text { hsCRP, } \\
\text { IL-6 }\end{array}$ \\
\hline
\end{tabular}


Table 1. Cont.

\begin{tabular}{|c|c|c|c|c|c|c|c|c|}
\hline $\begin{array}{l}\text { Esposito et al., } \\
2009 \text { [26] }\end{array}$ & $\begin{array}{l}195 ; \\
\text { olive oil: } 50 \text {, } \\
\text { control: } 51.5\end{array}$ & n.d. & n.d. & $\begin{array}{c}208, \\
\text { parallel }\end{array}$ & $\begin{array}{l}\text { Overweight, newly } \\
\text { diagnosed type } 2 \\
\text { diabetes mellitus, no } \\
\text { medication }\end{array}$ & $\begin{array}{c}\text { MedD + } \\
\text { 30-50 g olive } \\
\text { oil/day }\end{array}$ & Low-fat diet & adiponectin \\
\hline $\begin{array}{c}\text { Flynn et al., } 2010 \\
\text { [27] }\end{array}$ & $\begin{array}{l}28 \\
\text { n.d. }\end{array}$ & $59.2 \pm 6.1$ & $27.9 \pm 2.8$ & $\begin{array}{l}8 \text { weight } \\
\text { loss, } 24 \\
\text { follow-up, } \\
\text { cross-over }\end{array}$ & $\begin{array}{l}\text { Overweight women } \\
\text { with invasive breast } \\
\text { cancer, stable } \\
\text { medication }\end{array}$ & $\begin{array}{l}\text { Plant-based } \\
\text { olive oil diet } \\
\text { with at least } 3 \\
\text { tablespoons } \\
\text { extra virgin } \\
\text { olive oil/day }\end{array}$ & $\begin{array}{l}\text { National Cancer } \\
\text { Institute diet with } \\
\text { canola oil }\end{array}$ & CRP \\
\hline $\begin{array}{l}\text { Fuentes et al., } \\
2008 \text { [28] }\end{array}$ & $\begin{array}{l}20 \\
\text { n.d. }\end{array}$ & 23.3. \pm 1.5 & $24.65 \pm 2.91$ & $\begin{array}{c}4, \\
\text { cross-over }\end{array}$ & $\begin{array}{l}\text { Healthy, no } \\
\text { medication, no } \\
\text { intensive sports }\end{array}$ & $\begin{array}{l}\text { MUFA diet } \\
\text { based on extra } \\
\text { virgin olive oil } \\
\text { (38\% fat) }\end{array}$ & $\begin{array}{l}\text { SFA diet; low-fat } n-3 \\
\text { enriched diet based on } \\
\alpha \text {-linoleic acid }\end{array}$ & $\begin{array}{l}\text { sVCAM-1, } \\
\text { sICAM-1 }\end{array}$ \\
\hline $\begin{array}{l}\text { Gammelmark } \\
\text { et al., } 2012 \text { [29] }\end{array}$ & $\begin{array}{l}50 ; \\
\text { olive oil: } 52 \text {, } \\
\text { control: } 52\end{array}$ & $\begin{array}{c}\text { Olive oil: } 58.0 \pm 17.4 \\
\text { control: } 55.4 \pm 9.5\end{array}$ & $\begin{array}{c}\text { Olive oil: } 30.8 \pm 4.2 \\
\text { control: } 29.5 \pm 3.3\end{array}$ & 6, parallel & $\begin{array}{l}\text { Abdominally obese, } \\
\text { women } \\
\text { postmenopausal, no } \\
\text { medication except } \\
\text { NSAIDs }\end{array}$ & $\begin{array}{l}2 \text { g/day olive oil } \\
\text { capsules }\end{array}$ & $\begin{array}{l}2 \text { g/day fish oil } \\
\text { capsules }\end{array}$ & $\begin{array}{l}\text { Adiponectin, } \\
\text { CRP, IL-6 }\end{array}$ \\
\hline $\begin{array}{l}\text { Konstantinidou } \\
\text { et al., } 2010 \text { [30] }\end{array}$ & $\begin{array}{l}\text { 90; } \\
\text { olive oil: } 67 \text {, } \\
\text { control: } 63\end{array}$ & $\begin{array}{l}\text { Olive oil: } 45 \pm 10 \\
\text { control: } 44 \pm 10\end{array}$ & $\begin{array}{l}\text { Olive oil: } 25 \pm 4 \\
\text { control: } 26 \pm 5\end{array}$ & 12 , parallel & Healthy & $\begin{array}{c}\text { MedD + virgin } \\
\text { olive oil } \\
\text { (phenolic } \\
\text { content } 328 \\
\mathrm{mg} / \mathrm{kg} \text { ) }\end{array}$ & $\begin{array}{l}\text { MedD + washed virgin } \\
\text { olive oil (phenolic } \\
\text { content } 55 \mathrm{mg} / \mathrm{kg}) \\
\text { habitual diet }\end{array}$ & sP-selectin \\
\hline
\end{tabular}


Table 1. Cont.

\begin{tabular}{|c|c|c|c|c|c|c|c|c|}
\hline $\begin{array}{l}\text { Kontogianni } \\
\text { et al., } 2013 \text { [31] }\end{array}$ & $\begin{array}{l}37 \\
78\end{array}$ & $25.6 \pm 5.9$ & $21.9 \pm 2.5$ & $\begin{array}{c}6, \\
\text { cross-over }\end{array}$ & $\begin{array}{l}\text { Healthy, no } \\
\text { medication, no } \\
\text { intensive sports }\end{array}$ & $\begin{array}{l}15 \mathrm{~mL} / \text { day extra } \\
\text { virgin olive oil }\end{array}$ & 15 mL/day flaxseed oil & $\begin{array}{l}\text { Adiponectin, } \\
\text { CRP, } \\
\text { TNF- } \alpha\end{array}$ \\
\hline $\begin{array}{l}\text { Maki et al., } \\
2009 \text { [32] }\end{array}$ & $\begin{array}{l}76 ; \\
\text { olive oil: } 80 \text {, } \\
\text { control: } 88\end{array}$ & $\begin{array}{l}\text { Olive oil: } 49.6 \pm 1.4 \\
\text { control: } 49.4 \pm 1.7\end{array}$ & $\begin{array}{l}\text { Olive oil: } 31.1 \pm 1.1 \\
\text { control: } 32.6 \pm 1.5\end{array}$ & 4, parallel & $\begin{array}{l}\text { Healthy, abdominally } \\
\text { obese }\end{array}$ & $\begin{array}{l}2 \mathrm{~g} / \text { day olive oil } \\
\text { capsules }\end{array}$ & $\begin{array}{c}2 \text { g/day krill oil } \\
\text { capsules; } \\
2 \text { g/day menhaden oil } \\
\text { capsules }\end{array}$ & CRP \\
\hline $\begin{array}{l}\text { Mena et al., } \\
2009 \text { [33] }\end{array}$ & $\begin{array}{l}\text { 106; } \\
\text { olive oil: } 84 \text {, } \\
\text { control: } 76\end{array}$ & $\begin{array}{l}\text { Olive oil: } 66 \pm 11 \\
\text { control: } 66 \pm 7\end{array}$ & $\begin{array}{l}\text { Olive oil: } 28.0 \pm 2.9 \\
\text { control: } 27.8 \pm 3.2\end{array}$ & 12 , parallel & $\begin{array}{l}\text { Type } 2 \text { diabetes } \\
\text { mellitus or } \geqslant 3 \mathrm{CVD} \\
\text { risk factors, ACE } \\
\text { inhibitors, diuretics, } \\
\text { anti-hypertensive } \\
\text { agents, statins, } \\
\text { lipid-lowering } \\
\text { agents, insulin, oral } \\
\text { glucose-lowering } \\
\text { agents, aspirin or } \\
\text { anti-platelet drugs }\end{array}$ & $\begin{array}{c}\text { MedD + virgin } \\
\text { olive oil } \\
(1 \mathrm{~L} / \text { week })\end{array}$ & $\begin{array}{l}\text { MedD + nuts } \\
(30 \mathrm{~g} / \text { day }) ; \\
\text { low-fat diet }\end{array}$ & $\begin{array}{l}\text { sE-selectin, } \\
\text { sP-selectin, } \\
\text { sVCAM-1, } \\
\text { sICAM-1, } \\
\text { IL-6, CRP }\end{array}$ \\
\hline $\begin{array}{l}\text { Mori et al., } \\
2003 \text { [34] }\end{array}$ & $\begin{array}{l}51 ; \\
\text { olive oil: } 25 \text {, } \\
\text { control: } 28\end{array}$ & $\begin{array}{c}\text { Olive oil: } 61.5 \pm 1.9 \\
\text { control: } 60.9 \pm 1.9\end{array}$ & $\begin{array}{c}\text { Olive oil: } 29.9 \pm 1.0 \\
\text { control: } 28.6 \pm 0.7\end{array}$ & 6 , parallel & $\begin{array}{l}\text { Hypertension, type } 2 \\
\text { diabetes mellitus, } \\
\text { anti-hypertensive } \\
\text { therapy, oral } \\
\text { glucose-lowering } \\
\text { agents but no insulin }\end{array}$ & $\begin{array}{l}4 \mathrm{~g} / \text { day olive oil } \\
\text { capsules }\end{array}$ & $\begin{array}{l}4 \text { g/day EPA capsules; } \\
4 \text { g/day DHA capsules }\end{array}$ & $\begin{array}{l}\text { CRP, IL-6, } \\
\text { TNF- } \alpha\end{array}$ \\
\hline $\begin{array}{l}\text { Perez-Martinez } \\
\text { et al., } 2007 \text { [2] }\end{array}$ & $\begin{array}{c}16 \\
0\end{array}$ & n.d. & n.d. & $\begin{array}{c}4, \\
\text { cross-over }\end{array}$ & $\begin{array}{l}\text { Healthy, no } \\
\text { medication, no } \\
\text { intensive sports }\end{array}$ & $\begin{array}{l}\text { MedD + virgin } \\
\text { olive oil }\end{array}$ & $\begin{array}{l}\text { SFA diet; diet high in } \\
\text { carbohydrates }\end{array}$ & VCAM-1 \\
\hline
\end{tabular}


Table 1. Cont.

\begin{tabular}{|c|c|c|c|c|c|c|c|c|}
\hline $\begin{array}{l}\text { Pfeuffer } \text { et al., } \\
2011 \text { [35] }\end{array}$ & $\begin{array}{c}85 \\
\text { olive oil: } 0 \text {, } \\
\text { control: } 0\end{array}$ & n.d. & $\begin{array}{c}\text { Conjugated linoleic } \\
\text { acid: } 28.3 \pm 2.3, \\
\text { safflower oil: } 28.2 \pm \\
2.0, \\
\text { heated safflower oil: } \\
28.9 \pm 2.6\end{array}$ & 4, parallel & $\begin{array}{l}\text { Overweight or obese, } \\
\text { metabolic syndrome, } \\
\text { coronary heart } \\
\text { disease }\end{array}$ & $\begin{array}{l}4.5 \mathrm{~g} / \text { day olive } \\
\text { oil capsules }\end{array}$ & $\begin{array}{l}4.5 \mathrm{~g} / \text { day conjugated } \\
\text { linoleic acid mixture } \\
\text { capsules; } 4.5 \mathrm{~g} / \mathrm{day} \\
\text { safflower oil capsules; } \\
4.5 \mathrm{~g} / \text { day heated } \\
\text { safflower oil capsules }\end{array}$ & $\begin{array}{c}\text { CRP, } \\
\text { sICAM, } \\
\text { sVCAM, } \\
\text { sE-selectin }\end{array}$ \\
\hline $\begin{array}{l}\text { Sanders et al., } \\
2011 \text { [18] }\end{array}$ & $\begin{array}{c}367 ; \\
\text { olive oil: } 61 \text {, } \\
\text { control: } 56\end{array}$ & $\begin{array}{l}\text { Olive oil: } 55 \\
\text { (range: } 54-57 \text { ), } \\
\text { control: } 55 \\
\text { (range: } 54-57 \text { ) }\end{array}$ & $\begin{array}{l}\text { Olive oil, males: } 27 \\
\text { (range: } 26-28 \text { ), olive } \\
\text { oil, females: } 26 \\
\text { (range: } 25-27), \\
\text { control, males: } 26 \\
\text { (range: } 25-27 \text { ), } \\
\text { control, females: } 25 \\
\text { (range: } 24-26 \text { ) }\end{array}$ & 52 , parallel & $\begin{array}{l}\text { No CVD, } \\
\text { medication: statins, } \\
\text { anti-hypertensive } \\
\text { medication, hormone } \\
\text { replacement therapy, } \\
\text { thyroxine }\end{array}$ & $\begin{array}{c}3 \mathrm{~g} / \text { day refined } \\
\text { olive oil } \\
\text { capsules }\end{array}$ & $\begin{array}{c}\text { 0.45/0.9/1.8 g/day EPA } \\
\text { + DHA capsules }\end{array}$ & $\begin{array}{l}\text { CRP, } \\
\text { FMD }\end{array}$ \\
\hline $\begin{array}{l}\text { Singhal et al., } \\
2013 \text { [36] }\end{array}$ & $\begin{array}{c}324 ; \\
\text { olive oil: } 60 \text {, } \\
\text { control: } 66\end{array}$ & $\begin{array}{c}\text { Olive oil: } 27.6 \pm 4.7 \\
\text { control: } 28.2 \pm 4.8\end{array}$ & $\begin{array}{l}\text { Olive oil: } 23.6 \pm 3.5 \\
\text { control: } 23.6 \pm 34.3\end{array}$ & 16 , parallel & $\begin{array}{l}\text { Healthy, no } \\
\text { medication }\end{array}$ & $\begin{array}{l}4 \mathrm{~g} / \text { day olive oil } \\
\text { capsules }\end{array}$ & $\begin{array}{c}1.6 \mathrm{~g} / \text { day DHA }+2.4 \\
\text { g/day carrier oil } \\
\text { capsules }\end{array}$ & $\begin{array}{l}\text { CRP, } \\
\text { FMD }\end{array}$ \\
\hline $\begin{array}{l}\text { Sofi et al., } \\
2010 \text { [37] }\end{array}$ & $\begin{array}{c}11 ; \\
\text { olive oil: } 0 \text {, } \\
\text { control: } 33\end{array}$ & $\begin{array}{l}\text { Olive oil: } 54 \\
\text { (range: } 42-70 \text { ), } \\
\text { control: } 55 \\
\text { (range: } 30-41 \text { ) }\end{array}$ & $\begin{array}{c}\text { Olive oil: } 29.3 \pm 3.9 \\
\text { control: } 29.3 \pm 4.1\end{array}$ & 52 , parallel & $\begin{array}{l}\text { Non-alcoholic fatty } \\
\text { liver disease }\end{array}$ & $\begin{array}{c}6.5 \mathrm{~mL} / \text { day } \\
\text { olive oil }\end{array}$ & $\begin{array}{c}6.5 \mathrm{~mL} / \text { day olive oil } \\
\text { enriched with } n-3 \\
\text { PUFA }\end{array}$ & adiponectin \\
\hline $\begin{array}{c}\text { Stirban et al., } \\
2010[38]\end{array}$ & $\begin{array}{l}34 \\
\text { n.d. }\end{array}$ & $56.8 \pm 8.3$ & $31.3 \pm 4.1$ & $\begin{array}{c}6 \\
\text { cross-over }\end{array}$ & $\begin{array}{l}\text { Type } 2 \text { diabetes } \\
\text { mellitus, aspirin, } \\
\text { angiotensin-converting } \\
\text { enzyme inhibitors, } \\
\text { angiotensin receptor } \\
\text { blockers, calcium } \\
\text { channel blockers, } \\
\text { B-blockers, diuretics, } \\
\text { statins }\end{array}$ & $\begin{array}{l}2 \mathrm{~g} / \text { day olive oil } \\
\text { capsules }\end{array}$ & $\begin{array}{c}2 \text { g/day } n-3 \text { PUFA } \\
\text { capsules }\end{array}$ & FMD \\
\hline
\end{tabular}


Table 1. Cont.

\begin{tabular}{|c|c|c|c|c|c|c|c|c|}
\hline $\begin{array}{c}\text { Taylor et al., } \\
2005 \text { [39] }\end{array}$ & $\begin{array}{c}40 \\
\text { olive oil: } 0 \text {, } \\
\text { control: } 0\end{array}$ & $\begin{array}{l}\text { Olive oil: } 47 \pm 8 \\
\text { control: } 45 \pm 6\end{array}$ & $\begin{array}{l}\text { Olive oil: } 33 \pm 3 \\
\text { control: } 33 \pm 3\end{array}$ & 12 , parallel & Overweight, healthy & $\begin{array}{l}4.5 \text { g/day olive } \\
\text { oil capsules }\end{array}$ & $\begin{array}{l}4.5 \mathrm{~g} / \text { day conjugated } \\
\text { linoleic acid mixture } \\
\text { capsules }\end{array}$ & $\begin{array}{l}\text { adiponectin, } \\
\text { CRP, } \\
\text { FMD, } \\
\text { TNF- } \alpha\end{array}$ \\
\hline $\begin{array}{c}\text { Theobald et al., } \\
2007 \text { [40] }\end{array}$ & $\begin{array}{l}39 \\
50\end{array}$ & $\begin{array}{l}\text { Male: } 51.1 \pm 7.4 \\
\text { female: } 46.2 \pm 4.9\end{array}$ & $\begin{array}{c}\text { Male: } 51.1 \pm 7.4, \\
\text { females: } 46.2 \pm 4.9\end{array}$ & $\begin{array}{c}12 \\
\text { cross-over }\end{array}$ & $\begin{array}{l}\text { Healthy, no } \\
\text { medication }\end{array}$ & $\begin{array}{l}1.5 \mathrm{~g} / \text { day } \\
\text { refined olive oil } \\
\text { capsules }\end{array}$ & $\begin{array}{c}0.7 \text { g/day DHA } \\
\text { capsules }\end{array}$ & $\begin{array}{l}\text { IL-6, CRP, } \\
\text { sE-Selectin }\end{array}$ \\
\hline $\begin{array}{c}\text { Tholstrup et al., } \\
2008 \text { [41] }\end{array}$ & $\begin{array}{l}69 ; \\
\text { olive oil: } 100 \text {, } \\
\text { control: } 100\end{array}$ & $\begin{array}{c}\text { Olive oil: } 59.9 \pm 4.9, \\
\text { control: } 62.3 \pm 5.0\end{array}$ & $\begin{array}{l}\text { Olive oil: } 25.5 \pm 3.3 \\
\text { control: } 25.6 \pm 3.1\end{array}$ & 16 , parallel & $\begin{array}{l}\text { Healthy, } \\
\text { postmenopausal } \\
\text { women, no } \\
\text { medication }\end{array}$ & $\begin{array}{l}5.5 \mathrm{~g} / \text { day olive } \\
\text { oil capsules }\end{array}$ & $\begin{array}{l}5.5 \mathrm{~g} / \text { day conjugated } \\
\text { linoleic acid mixture } \\
\text { capsules; } 5.5 \mathrm{~g} / \text { day } \\
\text { conjugated linoleic } \\
\text { acid milk capsules }\end{array}$ & $\begin{array}{c}\text { IL-6, } \\
\text { ICAM-1, } \\
\text { VCAM-1 }\end{array}$ \\
\hline $\begin{array}{c}\text { Thomazella et al., } \\
2011 \text { [42] }\end{array}$ & $\begin{array}{c}40 \text {; } \\
\text { olive oil: } 0 \text {, } \\
\text { control: } 0\end{array}$ & $\begin{array}{c}\text { Olive oil: } 55.0 \pm 4.6 \\
\text { control: } 54.6 \pm 5.0\end{array}$ & $\begin{array}{c}\text { Olive oil: } 26.5 \pm 1.9 \\
\text { control: } 26.3 \pm 2.5\end{array}$ & 12 , parallel & $\begin{array}{l}\geqslant 1 \text { coronary event, } \\
\text { clinical stablility, } \\
\text { aspirin, anti-platelet } \\
\text { drugs, statins } \\
\text { (+ezetimibe), } \\
\text { nitrates, ACE } \\
\text { inhibitors, } \beta \text { blockers }\end{array}$ & $\begin{array}{c}\text { MedD + extra } \\
\text { virgin olive oil } \\
(30 \mathrm{~mL} / \text { day })\end{array}$ & $\begin{array}{l}\text { Low-fat therapeutic } \\
\text { lifestyle changes diet }\end{array}$ & $\begin{array}{c}\text { CRP, } \\
\text { sICAM-1, } \\
\text { sVCAM-1, } \\
\text { FMD }\end{array}$ \\
\hline $\begin{array}{l}\text { Urpi-Sarda et al., } \\
\quad 2012 \text { [43] }\end{array}$ & $\begin{array}{l}516 \\
\text { n.d. }\end{array}$ & n.d. & n.d. & 52 , parallel & $\begin{array}{l}\text { Type } 2 \text { diabetes } \\
\text { mellitus or } \geqslant 3 \mathrm{CVD} \\
\text { risk factors }\end{array}$ & $\begin{array}{c}\text { MedD + virgin } \\
\text { olive oil } \\
(1 \mathrm{~L} / \text { week })\end{array}$ & $\begin{array}{c}\text { MedD + nuts; low-fat } \\
\text { diet }\end{array}$ & $\begin{array}{l}\text { ICAM-1, } \\
\text { IL-6 }\end{array}$ \\
\hline
\end{tabular}


Table 1. Cont.

\begin{tabular}{|c|c|c|c|c|c|c|c|c|}
\hline $\begin{array}{c}\text { Voon et al., } 2011 \\
\text { [44] }\end{array}$ & $\begin{array}{l}45 \\
80\end{array}$ & $30.1 \pm 8.3$ & $30.1 \pm 8.3$ & $\begin{array}{c}5, \\
\text { cross-over }\end{array}$ & healthy & $\begin{array}{l}\text { Olive oil }(2 / 3 \text { of } \\
\text { total fat })\end{array}$ & $\begin{array}{l}\text { Coconut oil }(2 / 3 \text { of } \\
\text { total fat }) ; \text { palm olein } \\
\text { (2/3 of total fat })\end{array}$ & $\begin{array}{l}\text { CRP, IL-6, } \\
\text { TNF- } \alpha\end{array}$ \\
\hline $\begin{array}{l}\text { Wong et al., } 2010 \\
\text { [45] }\end{array}$ & $\begin{array}{l}\text { 97; } \\
\text { olive oil: } 58 \text {, } \\
\text { control: } 53\end{array}$ & $\begin{array}{l}\text { Olive oil: } 59.0 \pm 8.3 \text {, } \\
\text { control: } 61.2 \pm 9.0\end{array}$ & $\begin{array}{c}\text { Olive oil: } 26.4 \pm 4.4, \\
\text { control: } 25.2 \pm 3.7\end{array}$ & 12 , parallel & $\begin{array}{l}\text { Type } 2 \text { diabetes } \\
\text { mellitus, no } \\
\text { cardiovascular } \\
\text { events, oral } \\
\text { glucose-lowering } \\
\text { agents/insulin }\end{array}$ & $\begin{array}{l}4 \text { g/day olive oil } \\
\text { capsules }\end{array}$ & $\begin{array}{l}4 \text { g/day fish oil } \\
\text { capsules }\end{array}$ & $\begin{array}{l}\text { CRP, } \\
\text { FMD }\end{array}$ \\
\hline $\begin{array}{l}\text { Woodman et al., } \\
2003 \text { [46] }\end{array}$ & $\begin{array}{l}59 ; \\
\text { olive oil: } 25 \text {, } \\
\text { control: } 28\end{array}$ & $\begin{array}{c}\text { Olive oil: } 61.5 \pm 7.6 \\
\text { control: } 60.9 \pm 8.2\end{array}$ & $\begin{array}{c}\text { Olive oil: } 29.9 \pm 4.0, \\
\text { control: } 30.6 \pm 3.1\end{array}$ & 6 , parallel & $\begin{array}{l}\text { Obese, type } 2 \\
\text { diabetes mellitus and } \\
\text { hypertension, oral } \\
\text { glucose-lowering } \\
\text { agents and treatment } \\
\text { for hypertension }\end{array}$ & $\begin{array}{l}4 \mathrm{~g} / \text { day olive oil } \\
\text { capsules }\end{array}$ & $\begin{array}{l}4 \text { g/day EPA capsules; } \\
4 \text { g/day DHA capsules }\end{array}$ & $\begin{array}{c}\text { FMD, } \\
\text { P-selectin }\end{array}$ \\
\hline
\end{tabular}

$\mathrm{ACE}$ = angiotensin converitng enzyme; $\mathrm{CRP}=\mathrm{C}$-reactive protein; $\mathrm{CVD}=$ cardiovascular disease; DHA = docosahexaenoic acid; EPA = eicosapentaenoic acid; FMD = flow-mediated dilatation; ICAM-1 = intercellular adhesion molecule-1; IL-6 = interleukin-6; MedD = Mediterranean diet; NSAIDs = non-steroidal anti-inflammatory drugs; PUFA = polyunsaturated fatty acids; TNF- $\alpha=$ tumor necrosis factor $\alpha$; VCAM- $1=$ vascular cell adhesion molecule- 1 . 


\subsection{Markers of Inflammation}

Olive oil interventions resulted in a significantly more pronounced decrease in CRP (MD: -0.64 $\mathrm{mg} / \mathrm{L},(95 \% \mathrm{CI}-0.96$ to -0.31$), p<0.0001, I^{2}=66 \%$ ) as compared to the respective controls (Figure 3 ). Likewise, decreases in IL-6 levels were significantly stronger in the olive oil intervention groups as compared to controls (MD: -0.29 (95\% CI -0.7 to -0.02 ), $p<0.04, I^{2}=62 \%$ ) (Figure 4). For both outcome parameters, the largest number of studies used capsules as a mode of application and compared olive oil with $n-3$ fatty acids. Regarding this subgroup, mean differences were $(-0.28$ (95\% CI -0.75 to 0.18$), p<0.24, I^{2}=0 \%$ ) for CRP and ( -0.01 (95\% CI -0.48 to 0.49$), p<0.97, I^{2}=0 \%$ ) for IL-6, respectively. Pooled estimates of effects size for all markers of inflammation are summarized in Table 2.

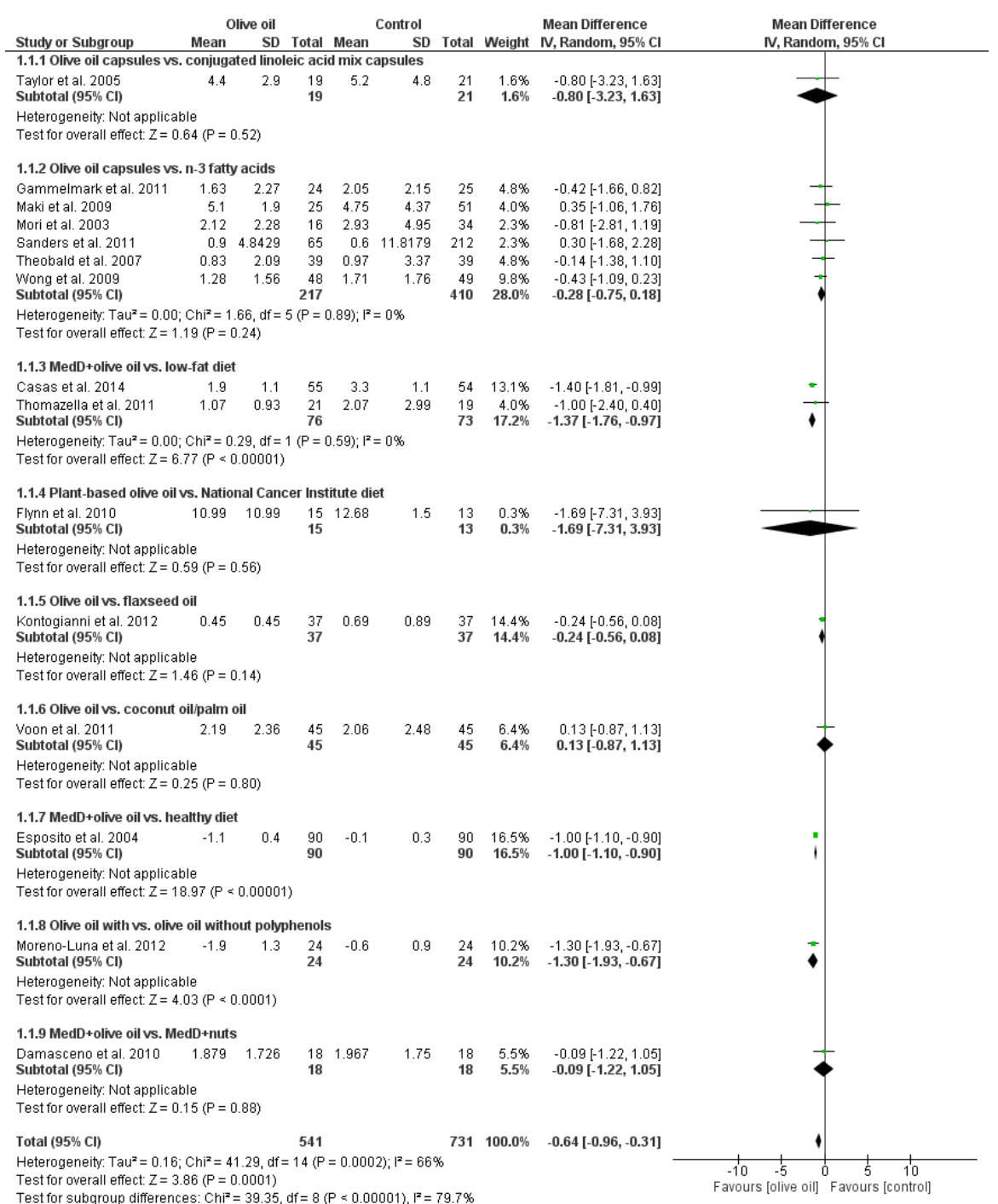

Figure 3. Effects of olive oil on C-reactive protein $(\mathrm{mg} / \mathrm{L})$. Forest plot showing pooled mean differences with $95 \%$ confidence intervals (CI) for 14 randomized controlled diets. For each study, the shaded square represents the point estimate of the intervention effect. The horizontal line joins the lower and upper limits of the $95 \%$ CI of these effects. The area of the shaded square reflects the relative weight of the study in the respective meta-analysis. The diamond at the bottom of the graph represents the pooled MD with the $95 \% \mathrm{CI}$ for all study groups. MedD = Mediterranean diet. 


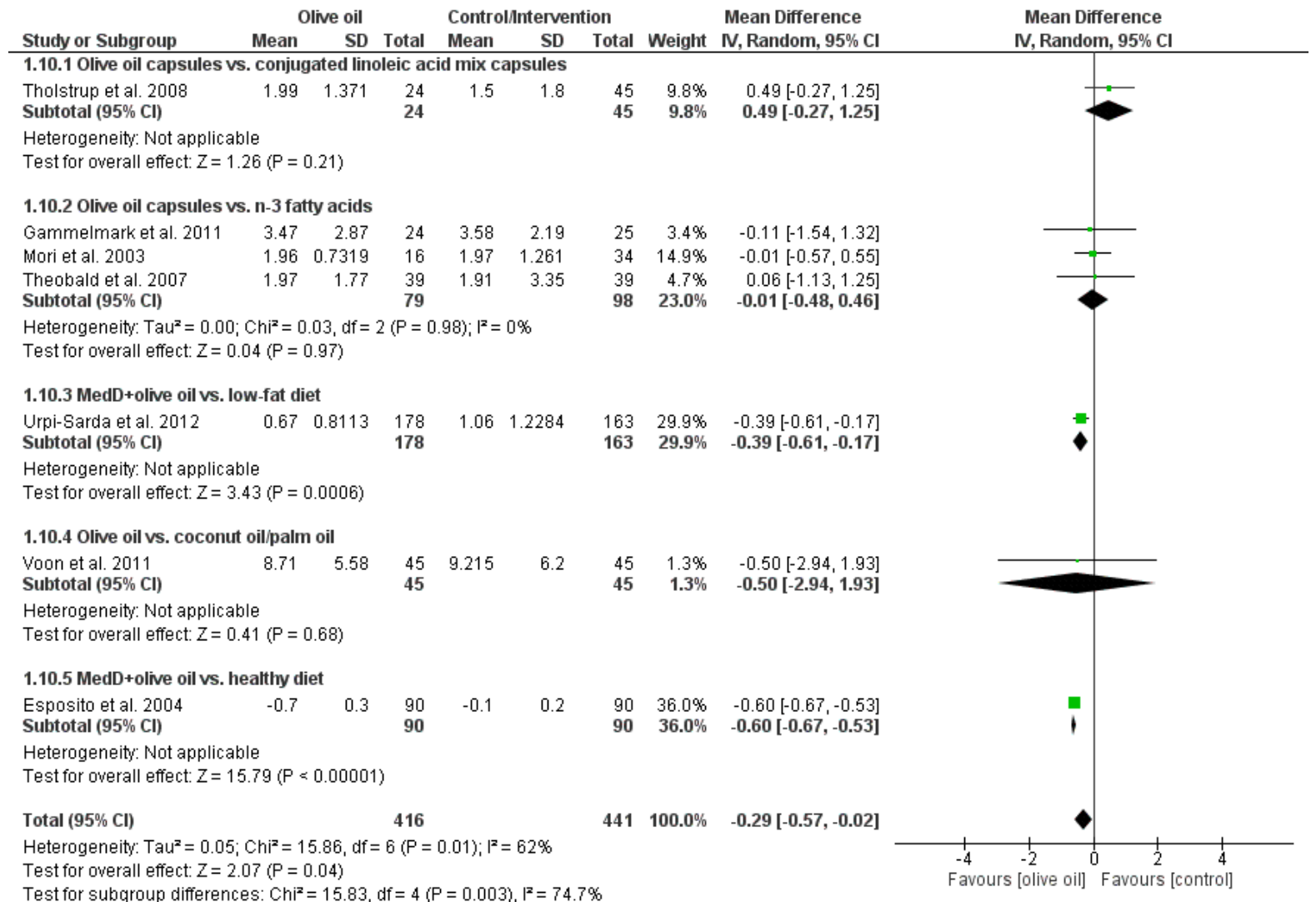

Figure 4. Effects of olive oil on interleukin-6 (pg/mL). Forest plot showing pooled mean differences with $95 \%$ confidence intervals (CI) for seven randomized controlled diets. For each study, the shaded square represents the point estimate of the intervention effect. The horizontal line joins the lower and upper limits of the $95 \% \mathrm{CI}$ of these effects. The area of the shaded square reflects the relative weight of the study in the respective meta-analysis. The diamond at the bottom of the graph represents the pooled MD with the 95\% CI for all study groups. MedD = Mediterranean diet.

FMD values (MD: 0.76 (95\% CI 0.27 to 1.24$), p<0.002, I^{2}=26 \%$ ) were more increased in individuals subjected to olive oil interventions (Figure 5). With respect to subgroups comparing olive oil with $n-3$, changes in FMD were significantly more pronounced in the olive oil groups when compared to their respective controls as well (MD: 0.63 (95\% CI 0.22 to 1.04), $p<0.003, I^{2}=0 \%$ ). Results for other markers of endothelial function are given in Table 2. 
Table 2. Pooled estimates of effect size for the results of olive oil interventions compared to respective controls.

\begin{tabular}{ccccccc}
\hline Outcome Parameter & $\begin{array}{c}\text { Mean } \\
\text { Difference }\end{array}$ & $\begin{array}{c}\mathbf{9 5 \%} \text { Confidence } \\
\text { Interval }\end{array}$ & $\boldsymbol{p}$-Value & $\begin{array}{c}\text { No. of } \\
\text { Studies }\end{array}$ & $\begin{array}{c}\text { Sample } \\
\text { Size }\end{array}$ & $\boldsymbol{I}^{\mathbf{2}}(\boldsymbol{\%})$ \\
\hline C-reactive protein (mg/L) & -0.64 & $(-0.96,-0.31)$ & 0.0001 & 15 & 1272 & 66 \\
Adiponectin $(\mathrm{mg} / \mathrm{L})$ & 0.44 & $(-0.20,1.09)$ & 0.18 & 6 & 313 & 56 \\
Interleukin-6 (pg/mL) & -0.29 & $(-0.57,-0.02)$ & 0.04 & 7 & 857 & 62 \\
Tumor necrosis factor- $\alpha(\mu \mathrm{g} / \mathrm{L})$ & 0.02 & $(-0.02,0.07)$ & 0.36 & 5 & 303 & 95 \\
sE-Selectin $(\mathrm{ng} / \mathrm{mL})$ & -3.16 & $(-4.07,-2.25)$ & 0.00001 & 2 & 187 & 0 \\
sP-Selectin $(\mathrm{ng} / \mathrm{mL})$ & 10.78 & $(4.01,17.54)$ & 0.002 & 4 & 358 & 41 \\
sICAM-1 (ng/L) & -0.02 & $(-0.04,0.00)$ & 0.02 & 7 & 724 & 84 \\
sVCAM-1 (ng/L) & -0.02 & $(-0.05,0.01)$ & 0.14 & 8 & 524 & 37 \\
FMD (\%) & 0.76 & $(0.27,1.24)$ & 0.002 & 8 & 851 & 26 \\
\hline
\end{tabular}

FMD = flow-mediated dilatation; $I^{2}=$ inconsistency (heterogeneity); ICAM-1 = Intercellular adhesion molecule-1; VCAM-1 = Vascular cell adhesion molecule-1.Markers of endothelial function.

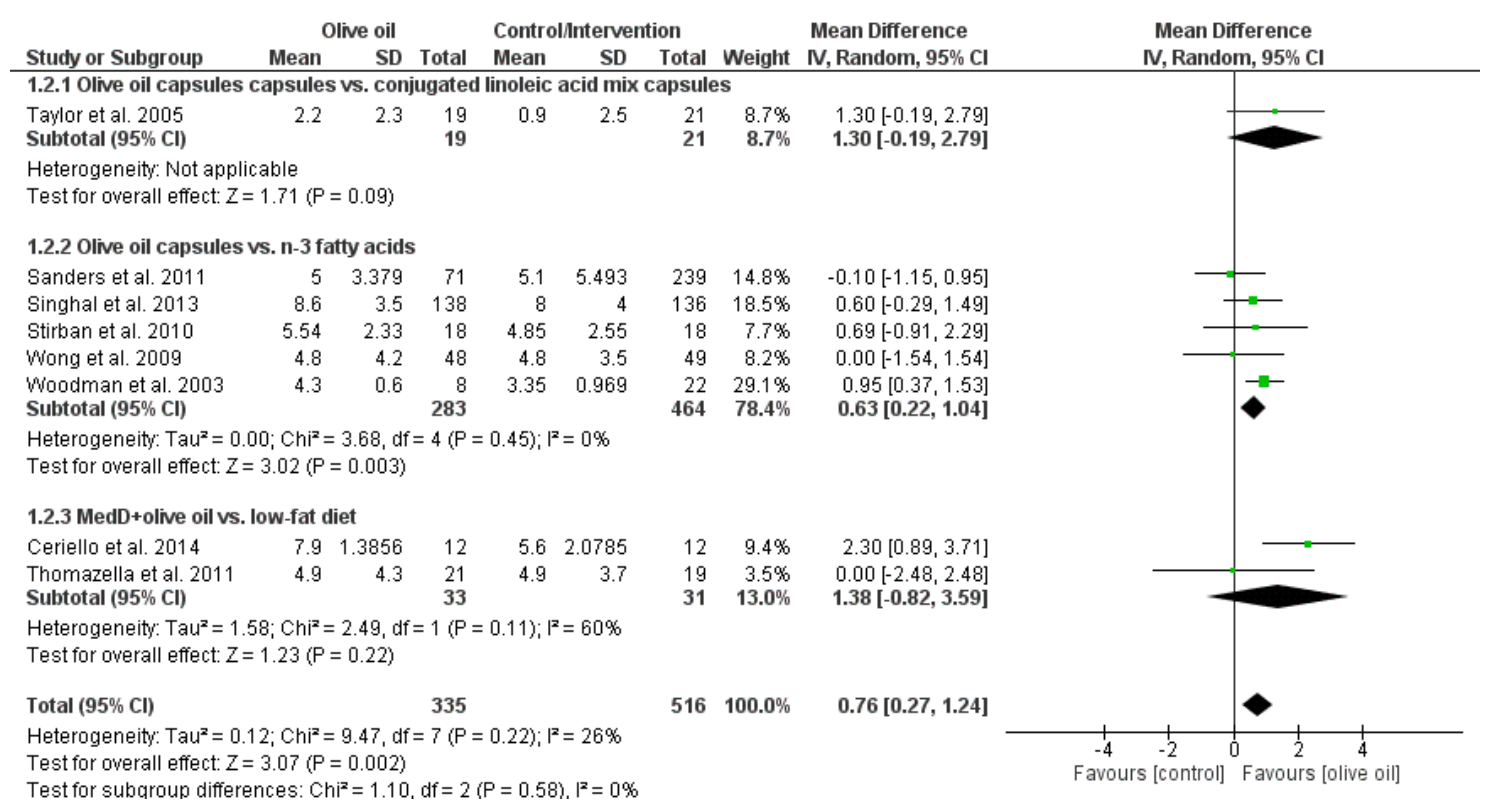

Figure 5. Effects of olive oil on flow-mediated dilatation (\%, absolute percentage). Forest plot showing pooled mean differences with $95 \%$ confidence intervals (CI) for eight randomized controlled diets. For each study, the shaded square represents the point estimate of the intervention effect. The horizontal line joins the lower and upper limits of the $95 \% \mathrm{CI}$ of these effects. The area of the shaded square reflects the relative weight of the study in the respective meta-analysis. The diamond at the bottom of the graph represents the pooled MD with the $95 \%$ CI for all study groups. Please note that the labeling of the X-axis has been switched as compared to Figures 3 and 4 respectively, since for flow-mediated dilatation an increase is considered to be favorable. MedD $=$ Mediterranean diet. 


\subsection{Publication Bias}

A funnel plot was generated for CRP (Figure 6), the only parameter assessed in at least 10 different trials. The plot indicates moderate asymmetry, thus a publication bias such as the difficulty in publishing negative or inconclusive data cannot be excluded to have an effect on the present meta-analyses.

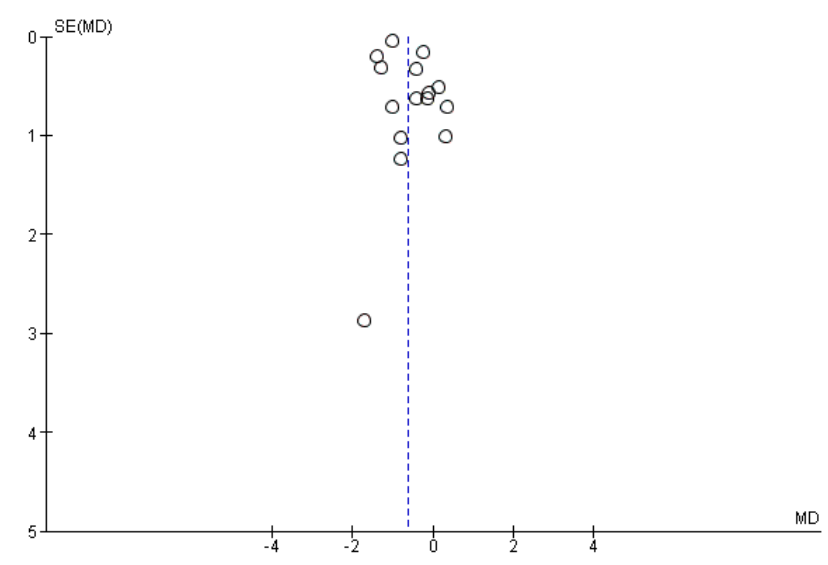

Figure 6. Funnel plot showing study precision against the mean differences effect estimate with $95 \%$ confidence intervals for $C$-reactive protein. $\mathrm{SE}=$ Standard error.

\section{Discussion}

Synthesis of data available from RCTs in the present systematic review suggest that markers of inflammation (CRP, IL-6) and those characterizing endothelial function (FMD, sE-Selectin) were favorably affected following interventions with olive oil. Although it is likely that no single biomarker is able to represent all the important risk information, most of the outcome parameters taken into consideration in this meta-analysis are regarded to be valid indicators of inflammation and endothelial dysfunction.

The association between serum CRP concentrations and cardiovascular risk has been suggested by various studies, and elevated CRP levels are regarded to be an independent risk factor for CVD [47,48]. The US Preventive Services Task Force performed a meta-analysis of 22 studies showing that CRP concentrations greater than $3.0 \mathrm{mg} / \mathrm{L}$ were associated with an approximate $60 \%$ excess risk of incident coronary heart disease as compared to levels less than $1 \mathrm{mg} / \mathrm{L}$ [49]. In addition, serum CRP levels can predict long-term risk of incidence of myocardial infarction, ischemic stroke, peripheral vascular disease and all-cause mortality [50]. Although the value of CRP as a predictor for CVD is still discussed controversially, decreases in CRP values found in the present meta-analysis may support the concept of a cardio-protective effect of olive oil intake. In addition to its function as a stimulator of CRP synthesis [51,52], IL-6 has been shown to correlate with an increased risk of coronary heart disease in prospective studies [53]. Moreover, increased baseline levels of IL-6 were found to predict future cardiovascular events [54].

The other inflammatory markers investigated in this systematic review were unaffected by olive oil interventions. Although $\mathrm{TNF}-\alpha$ is a relevant trigger during the inflammatory response, it has only rarely been assessed in epidemiological studies [55]. Likewise, data on a potential association between adiponectin and CVD risk are inconclusive [56-58]. An independent association of 
hypoadiponectinaemia with endothelial dysfunction measured by FMD has been observed by Tan et al. [59] in diabetic patients. Changes in TNF- $\alpha$ and adiponectin did not differ between olive oil interventions and respective controls in the present meta-analyses, which might be explained by the low number of study participants enrolled in the RCTs assessing these parameters.

To assess endothelial function, one of the standard non-invasive tools is FMD, which is regarded to reflect the local bioavailability of endothelium-derived vasoactive substances such as nitric oxide or endothelin-1. Reduced values of FMD are regarded to be early markers of atherosclerosis [60] as well as a predictor of future CVD events [8,61]. The association between reduced FMD and cardiovascular risk in individuals with varying baseline risk was demonstrated by several studies [62-64]. In a meta-analysis by Inaba and co-workers [8] synthesizing data of 5,500 participants of observational studies, each $1 \%$ reduction of FMD was associated with a $13 \%$ risk increase for cardiovascular events. This would equal an approximately $10 \%$ risk reduction given the effects of olive oil on FMD in the present meta-analysis.

Selectins are primary adhesion molecules in the inflammatory process expressed on the surfaces of activated endothelial cells, platelets, and leukocytes upon stimulation by TNF- $\alpha$, IL- 6 , and other pro-inflammatory cytokines [65]. Elevated concentrations of E-selectin were found to be associated with ischemic events independent of traditional risk markers in the PRIME study [66,67]. ICAM-1 and VCAM-1 promote the adhesion of leukocytes to the endothelium. They are both up-regulated by pro-inflammatory cytokines, although VCAM-1 is considered to be expressed in more advanced states of atherosclerosis. This might explain at least in part why, in contrast to ICAM-1, reductions in VCAM-1 levels were not significantly more pronounced following olive oil interventions in the present systematic review.

Vascular reactivity is affected by food intake. Atherosclerotic events may be slowed down by anti-oxidant compounds in food via limiting oxidative damage and restoring endothelial function [68]. Thus, polyphenol intake has been associated with low mortality rates caused by coronary heart disease [69]. Other studies indicate that endothelial function and lipid profile were improved by anti-oxidant and anti-inflammatory polyphenols [70]. Therefore, the phenolic compounds present in extra virgin olive oil might mediated the beneficial effects observed in the present meta-analyses. Extra virgin olive oil polyphenols demonstrated strong anti-oxidant properties in experimental studies $[3,13]$. In vivo studies in healthy volunteers and patients with hypercholesterolemia or stable coronary heart disease have demonstrated that polyphenols improve ischemic reactive hyperemia blood pressure as well as inflammatory status [4,71].

Another potential health-promoting ingredient of olive oil is oleic acid. High oleic acid content of olive oil was demonstrated to affect metabolic functions and cardiovascular risk factors [72]. In various meta-analyses and meta-regressions, beneficial effects of monounsaturated fatty acids such as oleic acid on cardiovascular risk factors have been reported although the data available at present are still ambiguous $[5,9,73]$.

\section{Limitations of the Systematic Review}

Although randomized controlled studies are regarded to prove a high level of evidence, the present systematic review has some major limitations as well. Various outcome parameters were associated with $\mathrm{I}^{2}$ values higher than $50 \%$, a threshold predetermined to indicate a considerable amount of heterogeneity. 
Trials varied with respect to study design, e.g., length of intervention, amount and type of olive oil used, classification of alternate source of fat ("control"), number of participants. Moreover, some study designs prescribed the intake of (extra virgin) olive oil provided by the investigators in pre-defined amounts (e.g., 30-50 g/day, $50 \mathrm{~mL} /$ day, or $1 \mathrm{~L} /$ week) to ensure a regular intake in combination with a MedD. However, besides vegetables, fruits, plant proteins, fish or whole grains, the traditional MedD is usually characterized by a high intake of olive oil in itself. Thus, these trials most likely have two separate sources of olive oil, which makes it impossible to quantify the absolute intake. Unfortunately, heterogeneous study protocols are a common characteristic in nutritional interventions. In addition, most studies failed to provide enough information for a thorough risk of bias analysis (Figure 1) and publication bias could only be assessed for one measured quantity, i.e., CRP. Some outcome parameters were collected in a rather low number of studies with a restricted sample size. This may at least in part explain another limitation of our study, i.e. the difficulty to model a link between biomarkers of inflammation and those of endothelial function via synthesizing the corresponding data from trials with heterogeneous designs. Taken together, these limitations implicate a cautious interpretation of the results of our meta-analyses.

\section{Conclusions}

The present systematic reviews provides some evidence that olive oil might exert beneficial effects on markers of inflammation and endothelial function. Since improvements in these parameters have been described in individuals adhering to a Mediterranean diet, olive oil might represent a key ingredient of this dietary pattern mediating these favorable effects.

\section{Author Contributions}

G.H. and L.S. developed the idea for this systematic review, L.S. prepared the protocol. Literature search was performed by L.S. and M.C., while data extraction, analyses, and synthesis was done by all authors. G.H. prepared the first draft of the manuscript. Disagreements were resolved by consensus, all authors read and approved of the final manuscript.

\section{Conflicts of Interest}

The authors declare no conflict of interest.

\section{References}

1. Lim, S.S.; Vos, T.; Flaxman, A.D.; Danaei, G.; Shibuya, K.; Adair-Rohani, H.; Amann, M.; Anderson, H.R.; Andrews, K.G.; Aryee, M.; et al. A comparative risk assessment of burden of disease and injury attributable to 67 risk factors and risk factor clusters in 21 regions, 1990-2010: A systematic analysis for the global burden of disease study 2010. Lancet 2012, 380, 2224-2260. [CrossRef] [PubMed]

2. Perez-Martinez, P.; Lopez-Miranda, J.; Blanco-Colio, L.; Bellido, C.; Jimenez, Y.; Moreno, J.A.; Delgado-Lista, J.; Egido, J.; Perez-Jimenez, F. The chronic intake of a mediterranean diet enriched in virgin olive oil, decreases nuclear transcription factor $\mathrm{kB}$ activation in peripheral blood mononuclear cells from healthy men. Atherosclerosis 2007, 194, e141-e146. [CrossRef] [PubMed] 
3. Sanchez-Fidalgo, S.; Sanchez de Ibarguen, L.; Cardeno, A.; Alarcon de la Lastra, C. Influence of extra virgin olive oil diet enriched with hydroxytyrosol in a chronic dss colitis model. Eur. J. Nutr. 2012, 51, 497-506. [CrossRef] [PubMed]

4. Moreno-Luna, R.; Muñoz-Hernandez, R.; Miranda, M.L.; Costa, A.F.; Jimenez-Jimenez, L.; Vallejo-Vaz, A.J.; Muriana, F.J.; Villar, J.; Stiefel, P. Olive oil polyphenols decrease blood pressure and improve endothelial function in young women with mild hypertension. Am. J. Hypertens. 2012, 25, 1299-1304. [CrossRef] [PubMed]

5. Schwingshackl, L.; Hoffmann, G. Monounsaturated fatty acids and risk of cardiovascular disease: Synopsis of the evidence available from systematic reviews and meta-analyses. Nutrients 2012, 4 , 1989-2007. [CrossRef] [PubMed]

6. Cook-Mills, J.M.; Marchese, M.E.; Abdala-Valencia, H. Vascular cell adhesion molecule-1 expression and signaling during disease: Regulation by reactive oxygen species and antioxidants. Antioxid. Redox Signal. 2011, 15, 1607-1638. [CrossRef] [PubMed]

7. Roos, M.W.; Sperber, G.O. A diffusion model of cerebral microischemia. Exp. Neurol. 1997, 147, 142-150. [CrossRef] [PubMed]

8. Inaba, Y.; Chen, J.A.; Bergmann, S.R. Prediction of future cardiovascular outcomes by flow-mediated vasodilatation of brachial artery: A meta-analysis. Int. J. Cardiovasc. Imaging 2010, 26, 631-640. [CrossRef] [PubMed]

9. Schwingshackl, L.; Hoffmann, G. Monounsaturated fatty acids, olive oil and health status: A systematic review and meta-analysis of cohort studies. Lipids Health Dis. 2014, 13, 154. [CrossRef] [PubMed]

10. Chrysohoou, C.; Panagiotakos, D.B.; Pitsavos, C.; Das, U.N.; Stefanadis, C. Adherence to the mediterranean diet attenuates inflammation and coagulation process in healthy adults: The Attica study. J. Am. Coll. Cardiol. 2004, 44, 152-158. [CrossRef] [PubMed]

11. Fung, T.T.; McCullough, M.L.; Newby, P.K.; Manson, J.E.; Meigs, J.B.; Rifai, N.; Willett, W.C.; Hu, F.B. Diet-quality scores and plasma concentrations of markers of inflammation and endothelial dysfunction. Am. J. Clin. Nutr. 2005, 82, 163-173. [PubMed]

12. Salas-Salvado, J.; Garcia-Arellano, A.; Estruch, R.; Marquez-Sandoval, F.; Corella, D.; Fiol, M.; Gomez-Gracia, E.; Vinoles, E.; Aros, F.; Herrera, C.; et al. Components of the mediterranean-type food pattern and serum inflammatory markers among patients at high risk for cardiovascular disease. Eur. J. Clin. Nutr. 2008, 62, 651-659. [CrossRef] [PubMed]

13. Martin, M.A.; Ramos, S.; Granado-Serrano, A.B.; Rodriguez-Ramiro, I.; Trujillo, M.; Bravo, L.; Goya, L. Hydroxytyrosol induces antioxidant/detoxificant enzymes and Nrf2 translocation via extracellular regulated kinases and phosphatidylinositol-3-kinase/protein kinase B pathways in HepG2 cells. Mol. Nutr. Food Res. 2010, 54, 956-966. [CrossRef] [PubMed]

14. International Prospective Register of Systematic Reviews. Available online: http://www.crd.york.ac.uk/ PROSPERO/ (accessed on 7 September 2015).

15. Higgins, J.P.; Altman, D.G.; Gøtzsche, P.C.; Jüni, P.; Moher, D.; Oxman, A.D.; Savovic, J.; Schulz, K.F.; Weeks, L.; Sterne, J.A.; et al. The cochrane collaboration's tool for assessing risk of bias in randomised trials. BMJ 2011, 343, d5928. [CrossRef] [PubMed] 
16. The Cochrane Collaboration. Avalaible online: http://tech.cochrane.org/revman/download (accessed on 7 September 2015).

17. Damasceno, N.R.; Perez-Heras, A.; Serra, M.; Cofan, M.; Sala-Vila, A.; Salas-Salvado, J.; Ros, E. Crossover study of diets enriched with virgin olive oil, walnuts or almonds. Effects on lipids and other cardiovascular risk markers. Nutr. Metab. Cardiovasc. Dis. 2011, 21 (Suppl. S1), S14-S20. [CrossRef] [PubMed]

18. Sanders, T.A.; Hall, W.L.; Maniou, Z.; Lewis, F.; Seed, P.T.; Chowienczyk, P.J. Effect of low doses of long-chain $n$-3 PUFAs on endothelial function and arterial stiffness: A randomized controlled trial. Am. J. Clin. Nutr. 2011, 94, 973-980. [CrossRef] [PubMed]

19. Casas, R.; Sacanella, E.; Urpi-Sarda, M.; Chiva-Blanch, G.; Ros, E.; Martinez-Gonzalez, M.A.; Covas, M.I.; Rosa Ma, L.R.; Salas-Salvado, J.; Fiol, M.; et al. The effects of the mediterranean diet on biomarkers of vascular wall inflammation and plaque vulnerability in subjects with high risk for cardiovascular disease. A randomized trial. PLoS ONE 2014, 9, e100084. [CrossRef] [PubMed]

20. Ceriello, A.; Esposito, K.; La Sala, L.; Pujadas, G.; De Nigris, V.; Testa, R.; Bucciarelli, L.; Rondinelli, M.; Genovese, S. The protective effect of the mediterranean diet on endothelial resistance to GLP-1 in type 2 diabetes: A preliminary report. Cardiovasc. Diabetol. 2014, 13, 140. [CrossRef] [PubMed]

21. Damsgaard, C.T.; Frøkiaer, H.; Andersen, A.D.; Lauritzen, L. Fish oil in combination with high or low intakes of linoleic acid lowers plasma triacylglycerols but does not affect other cardiovascular risk markers in healthy men. J. Nutr. 2008, 138, 1061-1066. [PubMed]

22. Damsgaard, C.T.; Lauritzen, L.; Calder, P.C.; Kjaer, T.R.; Frøkiaer, H. Reduced ex vivo interleukin-6 production by dietary fish oil is not modified by linoleic acid intake in healthy men. J. Nutr. 2009, 139, 1410-1414. [CrossRef] [PubMed]

23. Eschen, O.; Christensen, J.H.; de Caterina, R.; Schmidt, E.B. Soluble adhesion molecules in healthy subjects: A dose-response study using n-3 fatty acids. Nutr. Metab. Cardiovasc. Dis. 2004, 14, 180-185. [CrossRef]

24. Eschen, O.; Christensen, J.H.; LA Rovere, M.T.; Romano, P.; Sala, P.; Schmidt, E.B. Effects of marine $n$-3 fatty acids on circulating levels of soluble adhesion molecules in patients with chronic heart failure. Cell. Mol. Biol. 2010, 56, 45-51. [PubMed]

25. Esposito, K.; Marfella, R.; Ciotola, M.; di Palo, C.; Giugliano, F.; Giugliano, G.; D’Armiento, M.; D'Andrea, F.; Giugliano, D. Effect of a mediterranean-style diet on endothelial dysfunction and markers of vascular inflammation in the metabolic syndrome: A randomized trial. JAMA 2004, 292, 1440-1446. [CrossRef] [PubMed]

26. Esposito, K.; Maiorino, M.I.; Ciotola, M.; di Palo, C.; Scognamiglio, P.; Gicchino, M.; Petrizzo, M.; Saccomanno, F.; Beneduce, F.; Ceriello, A.; et al. Effects of a mediterranean-style diet on the need for antihyperglycemic drug therapy in patients with newly diagnosed type 2 diabetes: A randomized trial. Ann. Intern. Med. 2009, 151, 306-314. [CrossRef] [PubMed]

27. Flynn, M.M.; Reinert, S.E. Comparing an olive oil-enriched diet to a standard lower-fat diet for weight loss in breast cancer survivors: A pilot study. J. Women's Health 2010, 19, 1155-1161. [CrossRef] [PubMed] 
28. Fuentes, F.; Lopez-Miranda, J.; Perez-Martinez, P.; Jimenez, Y.; Marin, C.; Gomez, P.; Fernandez, J.M.; Caballero, J.; Delgado-Lista, J.; Perez-Jimenez, F. Chronic effects of a high-fat diet enriched with virgin olive oil and a low-fat diet enriched with $\alpha$-linolenic acid on postprandial endothelial function in healthy men. Br. J. Nutr. 2008, 100, 159-165. [CrossRef] [PubMed]

29. Gammelmark, A.; Madsen, T.; Varming, K.; Lundbye-Christensen, S.; Schmidt, E.B. Low-dose fish oil supplementation increases serum adiponectin without affecting inflammatory markers in overweight subjects. Nutr. Res. 2012, 32, 15-23. [CrossRef] [PubMed]

30. Konstantinidou, V.; Covas, M.I.; Munoz-Aguayo, D.; Khymenets, O.; de la Torre, R.; Saez, G.; Tormos Mdel, C.; Toledo, E.; Marti, A.; Ruiz-Gutierrez, V.; et al. In vivo nutrigenomic effects of virgin olive oil polyphenols within the frame of the mediterranean diet: A randomized controlled trial. FASEB J. Off. Publ. Fed. Am. Soc. Exp. Biol. 2010, 24, 2546-2557.

31. Kontogianni, M.D.; Vlassopoulos, A.; Gatzieva, A.; Farmaki, A.E.; Katsiougiannis, S.; Panagiotakos, D.B.; Kalogeropoulos, N.; Skopouli, F.N. Flaxseed oil does not affect inflammatory markers and lipid profile compared to olive oil, in young, healthy, normal weight adults. Metab. Clin. Exp. 2013, 62, 686-693. [CrossRef] [PubMed]

32. Maki, K.C.; Reeves, M.S.; Farmer, M.; Griinari, M.; Berge, K.; Vik, H.; Hubacher, R.; Rains, T.M. Krill oil supplementation increases plasma concentrations of eicosapentaenoic and docosahexaenoic acids in overweight and obese men and women. Nutr. Res. 2009, 29, 609-615. [CrossRef] [PubMed]

33. Mena, M.P.; Sacanella, E.; Vazquez-Agell, M.; Morales, M.; Fito, M.; Escoda, R.; Serrano-Martinez, M.; Salas-Salvado, J.; Benages, N.; Casas, R.; et al. Inhibition of circulating immune cell activation: A molecular antiinflammatory effect of the mediterranean diet. Am. J. Clin. Nutr. 2009, 89, 248-256. [CrossRef] [PubMed]

34. Mori, T.A.; Woodman, R.J.; Burke, V.; Puddey, I.B.; Croft, K.D.; Beilin, L.J. Effect of eicosapentaenoic acid and docosahexaenoic acid on oxidative stress and inflammatory markers in treated-hypertensive type 2 diabetic subjects. Free Radic. Biol. Med. 2003, 35, 772-781. [CrossRef]

35. Pfeuffer, M.; Fielitz, K.; Laue, C.; Winkler, P.; Rubin, D.; Helwig, U.; Giller, K.; Kammann, J.; Schwedhelm, E.; Böger, R.H.; et al. Cla does not impair endothelial function and decreases body weight as compared with safflower oil in overweight and obese male subjects. J. Am. Coll. Nutr. 2011, 30, 19-28. [CrossRef] [PubMed]

36. Singhal, A.; Lanigan, J.; Storry, C.; Low, S.; Birbara, T.; Lucas, A.; Deanfield, J. Docosahexaenoic acid supplementation, vascular function and risk factors for cardiovascular disease: A randomized controlled trial in young adults. J. Am.Heart Assoc. 2013, 2, e000283. [CrossRef] [PubMed]

37. Sofi, F.; Giangrandi, I.; Cesari, F.; Corsani, I.; Abbate, R.; Gensini, G.F.; Casini, A. Effects of a 1 -year dietary intervention with $n-3$ polyunsaturated fatty acid-enriched olive oil on non-alcoholic fatty liver disease patients: A preliminary study. Int. J. Food Sci. Nutr. 2010, 61, 792-802. [CrossRef] [PubMed]

38. Stirban, A.; Nandrean, S.; Götting, C.; Tamler, R.; Pop, A.; Negrean, M.; Gawlowski, T.; Stratmann, B.; Tschoepe, D. Effects of $n-3$ fatty acids on macro- and microvascular function in subjects with type 2 diabetes mellitus. Am. J. Clin. Nutr. 2010, 91, 808-813. [CrossRef] [PubMed] 
39. Taylor, J.S.; Williams, S.R.; Rhys, R.; James, P.; Frenneaux, M.P. Conjugated linoleic acid impairs endothelial function. Arterioscler. Thromb. Vasc. Biol. 2006, 26, 307-312. [CrossRef] [PubMed]

40. Theobald, H.E.; Goodall, A.H.; Sattar, N.; Talbot, D.C.; Chowienczyk, P.J.; Sanders, T.A. Low-dose docosahexaenoic acid lowers diastolic blood pressure in middle-aged men and women. J. Nutr. 2007, 137, 973-978. [PubMed]

41. Tholstrup, T.; Raff, M.; Straarup, E.M.; Lund, P.; Basu, S.; Bruun, J.M. An oil mixture with trans-10, cis-12 conjugated linoleic acid increases markers of inflammation and in vivo lipid peroxidation compared with cis-9, trans-11 conjugated linoleic acid in postmenopausal women. J. Nutr. 2008, 138, 1445-1451. [PubMed]

42. Thomazella, M.C.; Goes, M.F.; Andrade, C.R.; Debbas, V.; Barbeiro, D.F.; Correia, R.L.; Marie, S.K.; Cardounel, A.J.; daLuz, P.L.; Laurindo, F.R. Effects of high adherence to mediterranean or low-fat diets in medicated secondary prevention patients. Am. J. Cardiol. 2011, 108, 1523-1529. [CrossRef] [PubMed]

43. Urpi-Sarda, M.; Casas, R.; Chiva-Blanch, G.; Romero-Mamani, E.S.; Valderas-Martinez, P.; Salas-Salvado, J.; Covas, M.I.; Toledo, E.; Andres-Lacueva, C.; Llorach, R.; et al. The mediterranean diet pattern and its main components are associated with lower plasma concentrations of tumor necrosis factor receptor 60 in patients at high risk for cardiovascular disease. J. Nutr. 2012, 142, 1019-1025. [CrossRef] [PubMed]

44. Voon, P.T.; Ng, T.K.; Lee, V.K.; Nesaretnam, K. Diets high in palmitic acid (16:0), lauric and myristic acids $(12: 0+14: 0)$, or oleic acid $(18: 1)$ do not alter postprandial or fasting plasma homocysteine and inflammatory markers in healthy Malaysian adults. Am. J. Clin. Nutr. 2011, 94, 1451-1457. [CrossRef] [PubMed]

45. Wong, C.Y.; Yiu, K.H.; Li, S.W.; Lee, S.; Tam, S.; Lau, C.P.; Tse, H.F. Fish-oil supplement has neutral effects on vascular and metabolic function but improves renal function in patients with type 2 diabetes mellitus. Diabet. Med. J. Br. Diabet. Assoc. 2010, 27, 54-60. [CrossRef] [PubMed]

46. Woodman, R.J.; Mori, T.A.; Burke, V.; Puddey, I.B.; Barden, A.; Watts, G.F.; Beilin, L.J. Effects of purified eicosapentaenoic acid and docosahexaenoic acid on platelet, fibrinolytic and vascular function in hypertensive type 2 diabetic patients. Atherosclerosis 2003, 166, 85-93. [CrossRef]

47. Danesh, J.; Wheeler, J.G.; Hirschfield, G.M.; Eda, S.; Eiriksdottir, G.; Rumley, A.; Lowe, G.D.; Pepys, M.B.; Gudnason, V. $C$-reactive protein and other circulating markers of inflammation in the prediction of coronary heart disease. N. Engl. J. Med. 2004, 350, 1387-1397. [CrossRef] [PubMed]

48. Shah, T.; Casas, J.P.; Cooper, J.A.; Tzoulaki, I.; Sofat, R.; McCormack, V.; Smeeth, L.; Deanfield, J.E.; Lowe, G.D.; Rumley, A.; et al. Critical appraisal of crp measurement for the prediction of coronary heart disease events: New data and systematic review of 31 prospective cohorts. Int. J. Epidemiol. 2009, 38, 217-231. [CrossRef] [PubMed]

49. Buckley, D.I.; Fu, R.; Freeman, M.; Rogers, K.; Helfand, M. C-reactive protein as a risk factor for coronary heart disease: A systematic review and meta-analyses for the U.S. Preventive services task force. Ann. Intern. Med. 2009, 151, 483-495. [CrossRef] [PubMed]

50. Morrow, D.A.; Ridker, P.M. $C$-reactive protein, inflammation, and coronary risk. Med. Clin. N. Am. 2000, 84, 149-161. [CrossRef] 
51. Bataille, R.; Klein, B. $C$-reactive protein levels as a direct indicator of interleukin-6 levels in humans in vivo. Arthritis Rheum. 1992, 35, 982-984. [CrossRef] [PubMed]

52. Heinrich, P.C.; Castell, J.V.; Andus, T. Interleukin-6 and the acute phase response. Biochem. J. 1990, 265, 621-636. [CrossRef] [PubMed]

53. Danesh, J.; Kaptoge, S.; Mann, A.G.; Sarwar, N.; Wood, A.; Angleman, S.B.; Wensley, F.; Higgins, J.P.; Lennon, L.; Eiriksdottir, G.; et al. Long-term interleukin-6 levels and subsequent risk of coronary heart disease: Two new prospective studies and a systematic review. PLoS Med. 2008, 5, e78. [CrossRef] [PubMed]

54. Blake, G.J.; Ridker, P.M. Inflammatory bio-markers and cardiovascular risk prediction. J. Intern. Med. 2002, 252, 283-294. [CrossRef] [PubMed]

55. Kritchevsky, S.B.; Cesari, M.; Pahor, M. Inflammatory markers and cardiovascular health in older adults. Cardiovascul. Res. 2005, 66, 265-275. [CrossRef] [PubMed]

56. Hao, G.; Li, W.; Guo, R.; Yang, J.G.; Wang, Y.; Tian, Y.; Liu, M.Y.; Peng, Y.G.; Wang, Z.W. Serum total adiponectin level and the risk of cardiovascular disease in general population: A meta-analysis of 17 prospective studies. Atherosclerosis 2013, 228, 29-35. [CrossRef] [PubMed]

57. Kanhai, D.A.; Kranendonk, M.E.; Uiterwaal, C.S.; van der Graaf, Y.; Kappelle, L.J.; Visseren, F.L. Adiponectin and incident coronary heart disease and stroke. A systematic review and meta-analysis of prospective studies. Obes. Rev. 2013, 14, 555-567. [CrossRef] [PubMed]

58. Sook Lee, E.; Park, S.S.; Kim, E.; Sook Yoon, Y.; Ahn, H.Y.; Park, C.Y.; Ho Yun, Y.; Woo Oh, S. Association between adiponectin levels and coronary heart disease and mortality: A systematic review and meta-analysis. Int. J. Epidemiol. 2013, 42, 1029-1039. [CrossRef] [PubMed]

59. Tan, K.C.; Xu, A.; Chow, W.S.; Lam, M.C.; Ai, V.H.; Tam, S.C.; Lam, K.S. Hypoadiponectinemia is associated with impaired endothelium-dependent vasodilation. J. Clin. Endocrinol. Metab. 2004, 89, 765-769. [CrossRef] [PubMed]

60. Celermajer, D.S.; Sorensen, K.E.; Gooch, V.M.; Spiegelhalter, D.J.; Miller, O.I.; Sullivan, I.D.; Lloyd, J.K.; Deanfield, J.E. Non-invasive detection of endothelial dysfunction in children and adults at risk of atherosclerosis. Lancet 1992, 340, 1111-1115. [CrossRef]

61. Schroeder, S.; Enderle, M.D.; Ossen, R.; Meisner, C.; Baumbach, A.; Pfohl, M.; Herdeg, C.; Oberhoff, M.; Haering, H.U.; Karsch, K.R. Noninvasive determination of endothelium-mediated vasodilation as a screening test for coronary artery disease: Pilot study to assess the predictive value in comparison with angina pectoris, exercise electrocardiography, and myocardial perfusion imaging. Am. Heart J. 1999, 138, 731-739. [CrossRef]

62. Chan, S.Y.; Mancini, G.B.; Kuramoto, L.; Schulzer, M.; Frohlich, J.; Ignaszewski, A. The prognostic importance of endothelial dysfunction and carotid atheroma burden in patients with coronary artery disease. J. Am. Coll. Cardiol. 2003, 42, 1037-1043. [CrossRef]

63. Gokce, N.; Keaney, J.F.; Hunter, L.M.; Watkins, M.T.; Nedeljkovic, Z.S.; Menzoian, J.O.; Vita, J.A. Predictive value of noninvasively determined endothelial dysfunction for long-term cardiovascular events in patients with peripheral vascular disease. J. Am. Coll. Cardiol. 2003, 41, 1769-1775. [CrossRef]

64. Yeboah, J.; Folsom, A.R.; Burke, G.L.; Johnson, C.; Polak, J.F.; Post, W.; Lima, J.A.; Crouse, J.R.; Herrington, D.M. Predictive value of brachial flow-mediated dilation for incident cardiovascular 
events in a population-based study: The multi-ethnic study of atherosclerosis. Circulation 2009, 120, 502-509. [CrossRef] [PubMed]

65. Siemiatkowski, A.; Rogowski, F.; Wereszczyńska-Siemiatkowska, U.; Malinowska, L.; Borkowski, J. Soluble selectin profiles associated with severe trauma. Arch. Immunol. Ther. Exp. (Warsz.) 2001, 49, 317-324. [PubMed]

66. Cherian, P.; Hankey, G.J.; Eikelboom, J.W.; Thom, J.; Baker, R.I.; McQuillan, A.; Staton, J.; Yi, Q. Endothelial and platelet activation in acute ischemic stroke and its etiological subtypes. Stroke 2003, 34, 2132-2137. [CrossRef] [PubMed]

67. Prugger, C.; Luc, G.; Haas, B.; Morange, P.E.; Ferrieres, J.; Amouyel, P.; Kee, F.; Ducimetiere, P.; Empana, J.P.; Group, P.S. Multiple biomarkers for the prediction of ischemic stroke: The prime study. Arterioscler. Thromb. Vasc. Biol. 2013, 33, 659-666. [CrossRef] [PubMed]

68. Karatzi, K.N.; Papamichael, C.M.; Karatzis, E.N.; Papaioannou, T.G.; Aznaouridis, K.A.; Katsichti, P.P.; Stamatelopoulos, K.S.; Zampelas, A.; Lekakis, J.P.; Mavrikakis, M.E. Red wine acutely induces favorable effects on wave reflections and central pressures in coronary artery disease patients. Am. J. Hypertens. 2005, 18, 1161-1167. [CrossRef] [PubMed]

69. Hertog, M.G.; Kromhout, D.; Aravanis, C.; Blackburn, H.; Buzina, R.; Fidanza, F.; Giampaoli, S.; Jansen, A.; Menotti, A.; Nedeljkovic, S. Flavonoid intake and long-term risk of coronary heart disease and cancer in the seven countries study. Arch. Intern. Med. 1995, 155, 381-386. [CrossRef] [PubMed]

70. Zern, T.L.; Fernandez, M.L. Cardioprotective effects of dietary polyphenols. J. Nutr. 2005, 135, 2291-2294. [PubMed]

71. Ruano, J.; Lopez-Miranda, J.; Fuentes, F.; Moreno, J.A.; Bellido, C.; Perez-Martinez, P.; Lozano, A.; Gómez, P.; Jiménez, Y.; Pérez Jiménez, F. Phenolic content of virgin olive oil improves ischemic reactive hyperemia in hypercholesterolemic patients. J. Am. Coll. Cardiol. 2005, 46, 1864-1868. [CrossRef] [PubMed]

72. Bermudez, B.; Lopez, S.; Ortega, A.; Varela, L.M.; Pacheco, Y.M.; Abia, R.; Muriana, F.J. Oleic acid in olive oil: From a metabolic framework toward a clinical perspective. Curr. Pharm. Des. 2011, 17, 831-843. [CrossRef] [PubMed]

73. Martinez-Gonzalez, M.A.; Dominguez, L.J.; Delgado-Rodriguez, M. Olive oil consumption and risk of CHD and/or stroke: A meta-analysis of case-control, cohort and intervention studies. Br. J. Nutr. 2014, 112, 248-259. [CrossRef] [PubMed]

(C) 2015 by the authors; licensee MDPI, Basel, Switzerland. This article is an open access article distributed under the terms and conditions of the Creative Commons Attribution license (http://creativecommons.org/licenses/by/4.0/). 